\title{
Conformally invariant trilinear forms on the sphere
}

\author{
Jean-Louis Clerc and Bent Ørsted
}

January 4, 2010

\begin{abstract}
To each complex number $\lambda$ is associated a representation $\pi_{\lambda}$ of the conformal group $S O_{0}(1, n)$ on $\mathcal{C}^{\infty}\left(S^{n-1}\right)$ (spherical principal series). For three values $\lambda_{1}, \lambda_{2}, \lambda_{3}$, we construct a trilinear form on $\mathcal{C}^{\infty}\left(S^{n-1}\right) \times \mathcal{C}^{\infty}\left(S^{n-1}\right) \times \mathcal{C}^{\infty}\left(S^{n-1}\right)$, which is invariant by $\pi_{\lambda_{1}} \otimes \pi_{\lambda_{2}} \otimes$ $\pi_{\lambda_{3}}$. The trilinear form, first defined for $\left(\lambda_{1}, \lambda_{2}, \lambda_{3}\right)$ in an open set of $\mathbb{C}^{3}$ is extended meromorphically, with simple poles located in an explicit family of hyperplanes. For generic values of the parameters, we prove uniqueness of trilinear invariant forms.
\end{abstract}

${ }^{0} 2000$ Mathematics Subject Classification : 22E45, 43A85

\section{Introduction}

The motivation for this article came from the paper [1] by J. Bernstein and A. Reznikov. In order to estimate automorphic coefficients, they use trilinear invariant forms for $G=P G L_{2}(\mathbb{R})$. Their paper shows the importance of three related questions of harmonic analysis on $P G L_{2}(\mathbb{R})$ :

given three representations $\left(\pi_{1}, \mathcal{H}_{1}\right),\left(\pi_{2}, \mathcal{H}_{2}\right),\left(\pi_{3}, \mathcal{H}_{3}\right)$ in the principal spherical 1 series of the group $G$,

i) construct a trilinear invariant functional on $\mathcal{H}_{1} \times \mathcal{H}_{2} \times \mathcal{H}_{3}$

ii) prove uniqueness (up to a scalar) of such a trilinear invariant functional

iii) compute the value of the trilinear functional on the $K$ fixed vectors of $\mathcal{H}_{1}, \mathcal{H}_{2}, \mathcal{H}_{3}$ respectively.

The representations are realized on the unit circle, on which the group $G$ acts projectively, and indexed by a complex number. One possible generalization consists in replacing the unit circle by the $n-1$ dimensional sphere $S$, under the action of the conformal group $G=S O_{0}(1, n)$. For this case,

\footnotetext{
${ }^{1}$ with respect to the maximal compact subgroup $K=P O(2)$ of $G$
} 
we present here a construction of an invariant trilinear form (item $i$ )), which uses an analytic continuation over three complex parameters and discuss the uniqueness statement (item $i i)$ ) for generic values of the parameters. The computation of the normalization factor (item $i i i)$ ), even for more geometric situations, will be published elsewhere, (cf [3]).

In section 1 , we recall elementary facts about conformal geometry of the sphere (in particular we give a description of the orbits of $G$ in $S \times S \times S$ ), and introduce the representations which the paper is concerned with. In section 2, we define formally the trilinear invariant form, study the domain of convergence of the corresponding integral and determine the analytic continuation in the three complex parameters corresponding to the three representations. In section 3, we prove the uniqueness statement. The proof relies on Bruhat's theory, which we recall in an appendix at the end of the paper. In section 4 , we give an alternative approach to the construction of an invariant trilinear form, using a realization of the tensor product of two of the representations involved, thus making connection with [11].

The present paper only deals with regular values of the parameters. The residues at poles will yield new conformally invariant trilinear forms, supported by the singular orbits of $G$ in $S \times S \times S$ and involving differential operators akin the Yamabe operator on the sphere, worth of a further study. Other geometric situations are potential domains for similar results. Let $P_{1}, P_{2}, P_{3}$ be three parabolic subgroups of a semi-simple Lie group, such that $G$ acts on $G / P_{1} \times G / P_{2} \times G / P_{3}$ with a finite number of orbits (see [8], [10]). Take three representations induced by characters of $P_{1}, P_{2}, P_{3}$. Invariant trilinear forms for these three representations can plausibly be studied along the same lines as in the present paper. The case of three copies of the Shilov boundary $S$ of a bounded symmetric domain of tube type is specially appealing (see [4] for a description of the orbits of $G$ in $S \times S \times S)$.

Let us mention the paper by A. Deitmar [5], which has some overlap with our results. Previous work on the subject also includes [12] and [9].

The first author thanks D. Barlet and L. Bérard Bergery for conversations on various aspects of this paper and the Mathematics Department of Aarhus University for welcoming him during the preparation of the present work. 


\section{Conformal geometry of the sphere}

Let

$$
S=\mathbb{S}^{n-1}=\left\{x=\left(x_{1}, x_{2}, \ldots, x_{n}\right), \quad|x|^{2}=x_{1}^{2}+x_{2}^{2}+\cdots+x_{n}^{2}=1\right\}
$$

be the unit sphere in $\mathbb{R}^{n}$. We usually (and tacitly) assume $n \geq 3$, as the case $n=2$ needs a few minor changes, which are occasionally mentioned in the text. The group $K=S O(n)$ operates on $S$. Let

$$
\mathbf{1}^{+}=(1,0, \ldots, 0), \quad \mathbf{1}^{-}=(-1,0, \ldots, 0) .
$$

The stabilizer of $\mathbf{1}^{+}$in $K$ is the subgroup

$$
M \simeq S O(n-1)=\left\{\left(\begin{array}{ll}
1 & 0 \\
0 & u
\end{array}\right), u \in S O(n-1)\right\} .
$$

With this notation, $S \simeq K / M$ is a compact Riemannian symmetric space.

Another realization of the sphere is useful. Let $\mathbb{R}^{1, n}$ be the real vector space $\mathbb{R}^{n+1}$ with the quadratic form

$$
q(x)=[x, x]=x_{0}^{2}-\left(x_{1}^{2}+x_{2}^{2}+\cdots+x_{n}^{2}\right) .
$$

To $x=\left(x_{1}, x_{2}, \ldots, x_{n}\right)$ in $S$ associate $\widetilde{x}=\left(1, x_{1}, x_{2}, \ldots, x_{n}\right)$ in $\mathbb{R}^{1, n}$. The correspondance

$$
x \longmapsto \mathbb{R} \widetilde{x}
$$

associates to a point in $S$ an isotropic line in $\mathbb{R}^{1, n}$. The correspondance is easily seen to be bijective. The group $G=S O_{0}(1, n)$ acts naturally on the set of isotropic lines, and hence on $S$. Explicitly, for $x$ in $S$ and $g$ in $G, g(x)$ is the unique point in $S$ such that

$$
\widetilde{g(x)}=(g \widetilde{x})_{0}^{-1} g \widetilde{x} .
$$

For $x$ in $S$ and $g$ in $G$, set

$$
\kappa(g, x)=(g \widetilde{x})_{0}^{-1} .
$$

Let $x, y$ be in $S$. The following identity holds

$$
[\widetilde{x}, \widetilde{y}]=1-\langle x, y\rangle=\frac{1}{2}|x-y|^{2}
$$

so that for $g$ in $G$,

$$
|g(x)-g(y)|=\kappa(g, x)^{\frac{1}{2}}|x-y| \kappa(g, y)^{\frac{1}{2}} .
$$


The infinitesimal version of (4) is

$$
|D g(x) \xi|=\kappa(g, x)|\xi|
$$

for $\xi$ any tangent vector to $S$ at $x$, where $g$ is in $G$ and $D g(x)$ stands for the differential at $x$ of the map $x \mapsto g(x)$. Hence the action of $G$ on $S$ is conformal, and $\kappa(g, x)$ is interpreted as the conformal factor of $g$ at $x$.

We look at $K$ as a subgroup of $G$. It is a maximal compact subgroup of $G$. The stabilizer in $G$ of the point $\mathbf{1}^{+}$is the parabolic subgroup $P=M A N$, where

$$
A=\left\{a_{t}=\left(\begin{array}{ccccc}
\cosh t & \sinh t & 0 & \ldots & 0 \\
\sinh t & \cosh t & 0 & \ldots & 0 \\
0 & 0 & 1 & & \\
\vdots & \vdots & & \ddots & \\
0 & 0 & & & 1
\end{array}\right), \quad t \in \mathbb{R}\right\}
$$

and

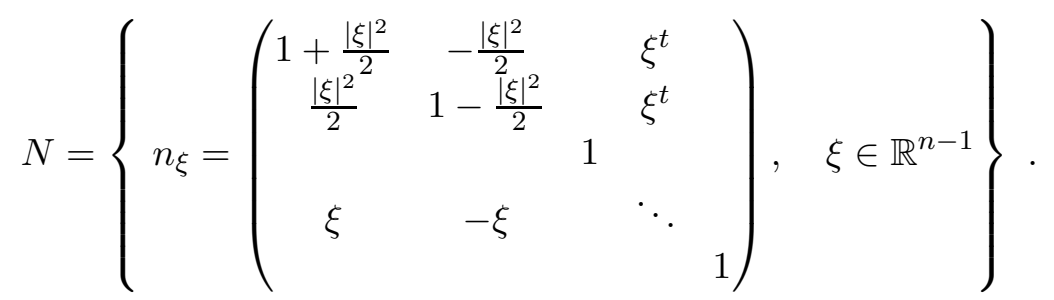

The element $a_{t}(t \in \mathbb{R})$ acts on $S$ by

$$
a_{t}\left(\begin{array}{c}
x_{1} \\
x_{2} \\
\ldots \\
x_{n}
\end{array}\right)=\left(\begin{array}{c}
\frac{\sinh t+x_{1} \cosh t}{\cosh t+x_{1} \sinh t} \\
\frac{x_{2}}{\cosh t+x_{1} \sinh t} \\
\vdots \\
\frac{x_{n}}{\cosh t+x_{1} \sinh t}
\end{array}\right) .
$$

Let $\bar{N}$ be the subgroup image of $N$ by the standard Cartan involution of $G\left(g \longmapsto\left(g^{t}\right)^{-1}\right)$ :

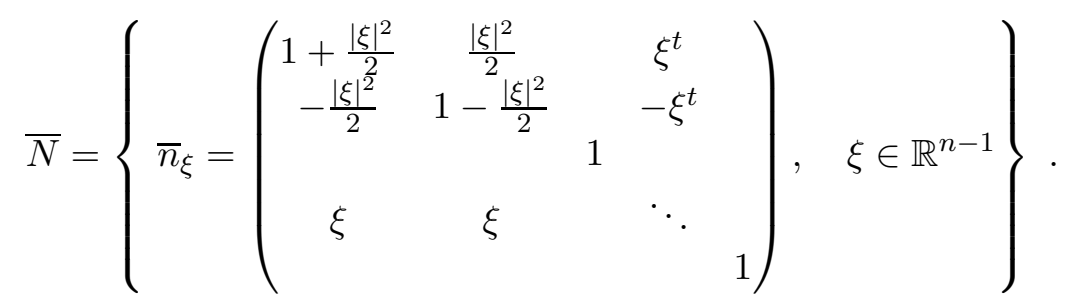


The map

$$
c: \xi \longmapsto \bar{n}_{\xi}\left(\mathbf{1}^{+}\right)=\left(\begin{array}{c}
\frac{1-|\xi|^{2}}{1+|\xi|^{2}} \\
\frac{2}{1+|\xi|^{2}} \xi
\end{array}\right) .
$$

is a diffeomorphism from $\mathbb{R}^{n-1}$ onto $S \backslash\left\{\mathbf{1}^{-}\right\}$. Its inverse is the classical stereographic projection from the source $1^{-}$onto the tangent space $T_{1}+S$ to $S$ at $\mathbf{1}^{+}$. When using this chart on $S$, we refer to the noncompact picture.

Proposition 1.1. The conformal factor $\kappa(g, x)$ is a smooth function of both $g$ and $x$, which satisfies the following properties :

i) $\forall g_{1}, g_{2} \in G, x \in S$,

$$
\kappa\left(g_{1} g_{2}, x\right)=\kappa\left(g_{1}, g_{2}(x)\right) \kappa\left(g_{2}, x\right)
$$

ii) $\forall g \in G, x \in S \quad \kappa\left(g, g^{-1}(x)\right)=\kappa\left(g^{-1}, x\right)^{-1}$

iii) $\forall x \in S, k \in K \quad \kappa(k, x)=1$

iv) $\forall x \in S, t \in \mathbb{R}, \quad \kappa\left(a_{t}, x\right)=\left(\cosh t+x_{1} \sinh t\right)^{-1}$.

Let $g$ in $G$. As the dimension of the tangent space $T_{x} S$ is $n-1$, the Jacobian of $g$ at $x$ is given by

$$
j(g, x)=\kappa(g, x)^{n-1} .
$$

The map $c: \mathbb{R}^{n-1} \longrightarrow S \backslash\{-\mathbf{1}\}$ is also conformal. In fact, one has the following relation, valid for any $\xi, \eta \in \mathbb{R}^{n-1}$ :

$$
|c(\xi)-c(\eta)|=\frac{2|\xi-\eta|}{\left(1+|\xi|^{2}\right)^{\frac{1}{2}}\left(1+|\eta|^{2}\right)^{\frac{1}{2}}},
$$

and its infinitesimal version

$$
|d c(\xi) \zeta|=\frac{2}{1+|\xi|^{2}}|\zeta|
$$

$\left(\zeta \in \mathbb{R}^{n-1}\right)$. The corresponding integration formula reads

$$
\int_{S} f(x) d \sigma(x)=\int_{\mathbb{R}^{n-1}} f(c(\xi)) \frac{2^{n-1}}{\left(1+|\xi|^{2}\right)^{n-1}} d \xi .
$$

Later, we will need a description of the orbits of $G$ in $S \times S \times S$ (for the diagonal action of $G$ ). Recall first that the group $G$ in its diagonal action on $S \times S$ has two orbits : 


$$
S_{\top}^{2}=\{(x, y) \in S \times S, x \neq y\}, \quad \Delta_{S}=\{(x, x), x \in S\} .
$$

As base-point in $S_{\top}^{2}$, choose $\left(\mathbf{1}^{+}, \mathbf{1}^{-}\right)$. The stabilizer of $\left(\mathbf{1}^{+}, \mathbf{1}^{-}\right)$in $G$ is the subgroup $M A$.

Proposition 1.2. Let $n \geq 3$. There are 5 orbits of $G$ in $S \times S \times S$, namely

$$
\begin{aligned}
& \mathcal{O}_{0}=\left\{\left(x_{1}, x_{2}, x_{3}\right), x_{i} \neq x_{j} \text { for } \mathrm{i} \neq \mathrm{j}\right\} \\
& \mathcal{O}_{1}=\left\{\left(x_{1}, x, x\right), x \neq x_{1}\right\} \\
& \mathcal{O}_{2}=\left\{\left(x, x_{2}, x\right), x \neq x_{2}\right\} \\
& \mathcal{O}_{3}=\left\{\left(x, x, x_{3}\right), x \neq x_{3}\right\} \\
& \mathcal{O}_{4}=\{(x, x, x)\} .
\end{aligned}
$$

Proof. The five subsets of $S \times S \times S$ are invariant under the diagonal action of $G$. So it suffices to show that $G$ is transitive on each of these sets.

- $G$ is transitive on $S$, hence on $\mathcal{O}_{4}$. Choose $\left(\mathbf{1}^{+}, \mathbf{1}^{+}, \mathbf{1}^{+}\right)$as base-point. The stabilizer of the base-point in $G$ is the subgroup $P=M A N$.

- The stabilizer $P$ of $\mathbf{1}^{+}$is transitive on $S \backslash\left\{\mathbf{1}^{+}\right\}$(the action of $N$ is already transitive on $S \backslash\left\{\mathbf{1}^{+}\right\}$), so that $G$ is transitive on $\mathcal{O}_{j}$, for $j=1,2,3$. As base-point in $\mathcal{O}_{3}$ (similar choices can be made for $\mathcal{O}_{1}$ and $\mathcal{O}_{2}$ ) choose $\left(\mathbf{1}^{+}, \mathbf{1}^{+}, \mathbf{1}^{-}\right)$. The stabilizer of $\left(\mathbf{1}^{+}, \mathbf{1}^{+}, \mathbf{1}^{-}\right)$is the subgroup $M A$.

- Let $x_{1}, x_{2}, x_{3}$ be in $\mathcal{O}_{0}$. We may assume w.l.o.g. that $x_{1}=\mathbf{1}^{+}, x_{2}=\mathbf{1}^{-}$ and $x_{3} \neq \mathbf{1}^{ \pm}$. The stabilizer of $\left(\mathbf{1}^{+}, \mathbf{1}^{-}\right)$in $G$ is $M A$. The orthogonal projection of $x_{3}$ on the hyperplane orthogonal to $\left(\mathbf{1}^{+}, \mathbf{1}^{-}\right)$is not 0 and can be mapped by $M$ to $c e_{2}$, with $0<c<1$, so that there exists $t \in \mathbb{R}$ such that $x_{3}$ is conjugate under $M$ to the point $\left(\tanh t, \frac{1}{\cosh t}, 0, \ldots, 0\right)=a_{t}\left(e_{2}\right)$. Hence any triplet in $\mathcal{O}_{0}$ is conjugate under $G$ to the triplet $\left(\mathbf{1}^{+}, \mathbf{1}^{-}, e_{2}\right)$. Its stabilizer in $G$ is the compact subgroup $\left\{k \in M, k e_{2}=e_{2}\right\} \simeq S O(n-2)$.

When $n=2$ ( $S$ is the unit circle), then there are two open orbits in $S \times S \times S$ unde the action of $S O_{0}(1,2)$, each characterized by the value of the orientation index of the three points in $S$. It is possible to remedy to this fact by using the slightly larger (no longer connected) group $O(1,2)$ instead of $S O_{0}(1,2)$.

Let $d \sigma$ be the Lebesgue measure on $S$ and let $\omega_{n-1}=\int_{S} d \sigma(x)$. Also set $\rho=\frac{n-1}{2}$. Under the action of $G$, the measure is $d \sigma$ is transformed according to

$$
\int_{S} f\left(g^{-1}(x)\right) d \sigma(x)=\int_{S} f(y) \kappa(g, y)^{2 \rho} d \sigma(y)
$$


Let $\lambda$ be in $\mathbb{C}$. For $f$ in $\mathcal{C}^{\infty}(S)$, the formula

$$
\pi_{\lambda}(g) f(x)=\kappa\left(g^{-1}, x\right)^{\rho+\lambda} f\left(g^{-1}(x)\right)
$$

defines a representation of the group $G$, which will be denoted by $\pi_{\lambda}$. It is a continous representation when the space $\mathcal{C}^{\infty}(S)$ is equipped with its natural Fréchet topology (see [14] for a systematic study of these representations).

The representations $\pi_{\lambda}$ and $\pi_{-\lambda}$ are dual in the sense that, for all $\varphi, \psi \in$ $\mathcal{C}^{\infty}(S)$

$$
\int_{S} \pi_{-\lambda}(g) \varphi(s) \pi_{\lambda}(g) \psi(s) d s=\int_{S} \varphi(s) \psi(s) d s
$$

as can be deduced from the change of variable formula (11). For $\lambda$ pure imaginary, the representation $\pi_{\lambda}$ can be extended continuously to $L^{2}(S)$ to yield a unitary representation of $G$ (this is the reason for using $\rho+\lambda$ in the definition of $\pi_{\lambda}$ ). Observe that the action of $K$ is independant of $\lambda$ and the constant fonction $\mathbb{I}_{S}$ (equal to 1 evereywhere) is fixed by the action of $K$.

For $\alpha$ in $\mathbb{C}$, let $k_{\alpha}$ be the kernel on $S \times S$ defined by

$$
k_{\alpha}(x, y)=|x-y|^{-\rho+\alpha} .
$$

It satisfies the following transformation property under the action of an element $g$ of $G$ :

$$
k_{\alpha}(g(x), g(y))=\kappa(g, x)^{-\frac{\rho}{2}+\frac{\alpha}{2}} k_{\alpha}(x, y) \kappa(g, y)^{-\frac{\rho}{2}+\frac{\alpha}{2}}
$$

for all $x, y$ in $S$.

\section{Construction of an invariant trilinear form}

\subsection{Formal construction}

Let $\lambda_{1}, \lambda_{2}, \lambda_{3}$ be three complex numbers. Let $\mathcal{T}$ be a continuous trilinear form from $\mathcal{C}^{\infty}(S) \times \mathcal{C}^{\infty}(S) \times \mathcal{C}^{\infty}(S)$ into $\mathbb{C}$. The functional $\mathcal{T}$ is said to be invariant w.r.t. $\pi_{\lambda_{1}}, \pi_{\lambda_{2}}, \pi_{\lambda_{3}}$ if, for every $f_{1}, f_{2}, f_{3}$ in $\mathcal{C}^{\infty}(S)$, and every $g$ in $G$

$$
\mathcal{T}\left(\pi_{\lambda_{1}}(g) f_{1}, \pi_{\lambda_{2}}(g) f_{2}, \pi_{\lambda_{3}}(g) f_{3}\right)=\mathcal{T}\left(f_{1}, f_{2}, f_{3}\right) .
$$

By Schwartz's kernel theorem, there exists a unique distribution $T$ on $S \times S \times S$, such that

$$
\mathcal{T}\left(f_{1}, f_{2}, f_{3}\right)=T\left(f_{1} \otimes f_{2} \otimes f_{3}\right)
$$


where, as usual, $f_{1} \otimes f_{2} \otimes f_{3}$ is the function on $S \times S \times S$ defined by

$$
\left(f_{1} \otimes f_{2} \otimes f_{3}\right)\left(x_{1}, x_{2}, x_{3}\right)=f_{1}\left(x_{1}\right) f_{2}\left(x_{2}\right) f_{3}\left(x_{3}\right) .
$$

Let $\alpha_{1}, \alpha_{2}, \alpha_{3}$ be complex numbers, and set $\boldsymbol{\alpha}=\left(\alpha_{1}, \alpha_{2}, \alpha_{3}\right)$. Let $K_{\boldsymbol{\alpha}}$ be the kernel on $S \times S \times S$ defined by

$$
K_{\boldsymbol{\alpha}}\left(x_{1}, x_{2}, x_{3}\right)=k_{\alpha_{1}}\left(x_{2}, x_{3}\right) k_{\alpha_{2}}\left(x_{3}, x_{1}\right) k_{\alpha_{3}}\left(x_{1}, x_{2}\right)
$$

For $f_{1}, f_{2}, f_{3}$ three functions in $\mathcal{C}^{\infty}(S)$, define the trilinear functional $\mathcal{K}_{\boldsymbol{\alpha}}$ by

$\mathcal{K}_{\boldsymbol{\alpha}}\left(f_{1}, f_{2}, f_{3}\right)=\int_{S \times S \times S} \underset{\boldsymbol{\alpha}}{K_{\boldsymbol{\alpha}}}\left(x_{1}, x_{2}, x_{3}\right) f_{1}\left(x_{1}\right) f_{2}\left(x_{2}\right) f_{3}\left(x_{3}\right) d \sigma\left(x_{1}\right) d \sigma\left(x_{2}\right) d \sigma\left(x_{3}\right)$

whenever it makes sense.

Theorem 2.1. Let $\boldsymbol{\lambda}=\left(\lambda_{1}, \lambda_{2}, \lambda_{3}\right) \in \mathbb{C}^{3}$. Define $\boldsymbol{\alpha}=\left(\alpha_{1}, \alpha_{2}, \alpha_{3}\right)$ by the relations

$$
\begin{aligned}
& \alpha_{1}=-\lambda_{1}+\lambda_{2}+\lambda_{3} \\
& \alpha_{2}=-\lambda_{2}+\lambda_{3}+\lambda_{1} \\
& \alpha_{3}=-\lambda_{3}+\lambda_{1}+\lambda_{2} .
\end{aligned}
$$

Then

$$
\mathcal{K}_{\boldsymbol{\alpha}}\left(\pi_{\lambda_{1}}(g) f_{1}, \pi_{\lambda_{2}}(g) f_{2}, \pi_{\lambda_{3}}(g) f_{3}\right)=\mathcal{K}_{\boldsymbol{\alpha}}\left(f_{1}, f_{2}, f_{3}\right),
$$

whenever the integral on the right handside is defined.

The proof is obtained by the change of variables $y_{j}=g^{-1}\left(x_{j}\right)(j=1,2,3)$ in the integral defining the left hand-side, using (14) and (11). Observe that the right-handside integral converges if, for $i \neq j$, the supports of $f_{i}$ and $f_{j}$ are disjoint, or if $\int_{S \times S \times S}\left|K_{\boldsymbol{\alpha}}\left(x_{1}, x_{2}, x_{3}\right)\right| d \sigma\left(x_{1}\right) d \sigma\left(x_{2}\right) d \sigma\left(x_{3}\right)<+\infty$.

The version of the trilinear functional in the noncompact picture will be useful. Let $\boldsymbol{\alpha}=\left(\alpha_{1}, \alpha_{2}, \alpha_{3}\right) \in \mathbb{C}^{3}$ and set for $y_{1}, y_{2}, y_{3} \in \mathbb{R}^{n-1}$

$$
J_{\boldsymbol{\alpha}}\left(y_{1}, y_{2}, y_{3}\right)=\left|y_{1}-y_{2}\right|^{-\rho+\alpha_{3}}\left|y_{2}-y_{3}\right|^{-\rho+\alpha_{1}}\left|y_{3}-y_{1}\right|^{-\rho+\alpha_{2}}
$$

and, whenever it makes sense, let $\mathcal{J}_{\boldsymbol{\alpha}}$ be the associated distribution on $\mathbb{R}^{n-1} \times \mathbb{R}^{n-1} \times \mathbb{R}^{n-1}$ given by

$$
\mathcal{J}_{\boldsymbol{\alpha}}(\varphi)=\int J_{\boldsymbol{\alpha}}\left(y_{1}, y_{2}, y_{3}\right) \varphi\left(y_{1}, y_{2}, y_{3}\right) d y_{1} d y_{2} d y_{3}
$$

$\left(\varphi \in \mathcal{C}^{\infty}\left(\mathbb{R}^{n-1} \times \mathbb{R}^{n-1} \times \mathbb{R}^{n-1}\right)\right)$. Moreover, let $\Psi_{\boldsymbol{\alpha}}$ be the function defined on $\mathbb{R}^{n-1} \times \mathbb{R}^{n-1} \times \mathbb{R}^{n-1}$ by

$\Psi_{\boldsymbol{\alpha}}\left(y_{1}, y_{2}, y_{3}\right)=\left(1+\left|y_{1}\right|^{2}\right)^{-\rho-\frac{\alpha_{2}}{2}-\frac{\alpha_{3}}{2}}\left(1+\left|y_{2}\right|^{2}\right)^{-\rho-\frac{\alpha_{3}}{2}-\frac{\alpha_{1}}{2}}\left(1+\left|y_{3}\right|^{2}\right)^{-\rho-\frac{\alpha_{1}}{2}-\frac{\alpha_{2}}{2}}$. 
Proposition 2.1. Let $f \in \mathcal{C}^{\infty}(S \times S \times S)$. Then

$$
\mathcal{K}_{\boldsymbol{\alpha}}(f)=2^{3(n-1)} \mathcal{J}_{\boldsymbol{\alpha}}\left((f \circ c) \Psi_{\boldsymbol{\alpha}}\right)
$$

whenever the left hand side is defined.

This is merely the change of variable (10) in the integral (18).

\section{$2.2 \quad$ Integrability of the kernel $K_{\alpha}$}

Theorem 2.2. The kernel $K_{\alpha}$ is integrable on $S \times S \times S$ if and only if

$$
\begin{aligned}
& \Re \alpha_{j}>-\rho, \quad j=1,2,3 \\
& \Re \alpha_{1}+\Re \alpha_{2}+\Re \alpha_{3}>-\rho
\end{aligned}
$$

Proof. It is enough to study the integral when the $\alpha$ 's are real, in which case the kernel $K_{\boldsymbol{\alpha}}$ is positive. Let $\mathcal{U}$ be a (small) neighborhood of $\left(\mathbf{1}^{+}, \mathbf{1}^{+}, \mathbf{1}^{+}\right)$ in $S \times S \times S$. Let $g$ be in $G$. As $x$ varies in $S, j(g, x)$ remains bounded from below and from above. Thanks to the transformation property of the kernel $k_{\alpha}$ (14), the integrals of $K_{\boldsymbol{\alpha}}$ over $\mathcal{U}$ and over $g(\mathcal{U})$ are of the same nature. As $\mathcal{U}$ meets all $G$-orbits, a partition of unity argument shows that the integrability over $S$ of the kernel $K_{\boldsymbol{\alpha}}$ is equivalent to its integrability

over $\mathcal{U}$. Now use the stereographic projection to see that the integrability of $K_{\boldsymbol{\alpha}}$ over $\mathcal{U}$ is equivalent to the integrability of $J_{\boldsymbol{\alpha}}$ over $c^{-1}(\mathcal{U})$, which is a (small) neighborhood of $(0,0,0)$ in $\mathbb{R}^{n-1} \times \mathbb{R}^{n-1} \times \mathbb{R}^{n-1}$.

Hence, we are reduced to study the convergence of the integral

$$
I_{1}=\int_{\substack{\left|\xi_{1}\right|<\delta \\\left|\xi_{2}\right|<\delta \\\left|\xi_{3}\right|<\delta}}\left|\xi_{1}-\xi_{2}\right|^{\alpha_{3}-\rho}\left|\xi_{2}-\xi_{3}\right|^{\alpha_{1}-\rho}\left|\xi_{3}-\xi_{1}\right|^{\alpha_{2}-\rho} d \xi_{1} d \xi_{2} d \xi_{3}
$$

where $\delta$ is a small positive number. Set

$$
y_{1}=\xi_{1}, \quad y_{2}=\xi_{1}-\xi_{2}, \quad y_{3}=-\xi_{2}+\xi_{3} .
$$

Then, after integrating with respect to $y_{1}$, the integral $I_{1}$ is seen to be of the same nature as the integral

$$
I_{2}=\int_{\substack{\left|y_{2}\right|<\delta \\\left|y_{3}\right|<\delta}}\left|y_{2}\right|^{\alpha_{3}-\rho}\left|y_{3}\right|^{\alpha_{2}-\rho}\left|y_{2}-y_{3}\right|^{\alpha_{1}-\rho} d y_{2} d y_{3} .
$$

Let $\Sigma$ be the unit ball in $\mathbb{R}^{(n-1)} \times \mathbb{R}^{(n-1)}$, and let $d \boldsymbol{\sigma}$ be the Lebesgue measure on $\Sigma$. Use polar coordinates $\left(r,\left(\sigma_{2}, \sigma_{3}\right)\right)$ defined by

$$
y_{2}=r \sigma_{2}, y_{3}=r \sigma_{3}, \quad r^{2}=\left|y_{2}\right|^{2}+\left|y_{3}\right|^{2}, \quad\left|\sigma_{1}\right|^{2}+\left|\sigma_{3}\right|^{2}=1
$$


to obtain that $I_{2}$ is of the same nature as

$$
I_{3}=\int_{0}^{\delta} r^{\alpha_{1}+\alpha_{2}+\alpha_{3}-3 \rho+2(n-1)-1} d r \times \int_{\Sigma}\left|\sigma_{2}\right|^{\alpha_{3}-\rho}\left|\sigma_{3}\right|^{\alpha_{2}-\rho}\left|\sigma_{2}-\sigma_{3}\right|^{\alpha_{1}-\rho} d \boldsymbol{\sigma} .
$$

The first factor converges if and only if condition (25) is satisfied. It remains to discuss the convergence of

$$
I_{4}=\int_{\Sigma}\left|\sigma_{2}\right|^{\alpha_{3}-\rho}\left|\sigma_{3}\right|^{\alpha_{2}-\rho}\left|\sigma_{2}-\sigma_{3}\right|^{\alpha_{1}-\rho} d \boldsymbol{\sigma} .
$$

Let $\delta>0$ and consider the following open subsets of $\Sigma$ :

$\Sigma_{2}=\left\{\left(\sigma_{2}, \sigma_{3}\right),\left|\sigma_{2}\right|<\delta\right\}, \Sigma_{3}=\left\{\left(\sigma_{2}, \sigma_{3}\right),\left|\sigma_{3}\right|<\delta\right\}, \Sigma_{1}=\left\{\left(\sigma_{2}, \sigma_{3}\right),\left|\sigma_{2}-\sigma_{3}\right|<\delta\right\}$.

For $\delta$ small enough, these sets are disjoint (recall that $\left|\sigma_{2}\right|^{2}+\left|\sigma_{3}\right|^{2}=1$ ). On $\Sigma \backslash\left(\Sigma_{1} \cup \Sigma_{2} \cup \Sigma_{3}\right)$ the function to be integrated is bounded from below. Hence the integral $I_{4}$ is convergent if and only the integrals

$$
K_{j}=\int_{\Sigma_{j}}\left|\sigma_{2}\right|^{\alpha_{3}-\rho}\left|\sigma_{3}\right|^{\alpha_{2}-\rho}\left|\sigma_{2}-\sigma_{3}\right|^{\alpha_{1}-\rho} d \boldsymbol{\sigma}
$$

are convergent for $j=1,2,3$. Let $j=2$. On $\Sigma_{2}$, both $\left|\sigma_{3}\right|$ and $\left|\sigma_{2}-\sigma_{3}\right|$ are bounded from below, so that it is equivalent to study the convergence of the integral

$$
\int_{\Sigma_{2}}\left|\sigma_{2}\right|^{\alpha_{3}-\rho} d \boldsymbol{\sigma}
$$

We are reduced to a classical situation and $K_{2}$ converges if and only if $\alpha_{3}-\rho>-(n-1)$. A similar study applies to $K_{1}$ and $K_{3}$. This completes the proof of Theorem 2.2 ,

Corollary 2.1. Let $\lambda_{1}, \lambda_{2}, \lambda_{3}$ be three complex numbers satisfying the condition

$$
0 \leq \Re\left(\lambda_{j}\right)<\rho .
$$

Define $\boldsymbol{\alpha}$ by the relations (19). Then the kernel $K_{\boldsymbol{\alpha}}$ is integrable, and the corresponding trilinear form is invariant for $\pi_{\lambda_{1}}, \pi_{\lambda_{2}}, \pi_{\lambda_{3}}$.

Proof. The conditions on $\boldsymbol{\lambda}=\left(\lambda_{1}, \lambda_{2}, \lambda_{3}\right)$ imply that $\Re\left(\alpha_{j}\right)>-\rho$ for $j=$ $1,2,3$ and $\Re\left(\alpha_{1}+\alpha_{2}+\alpha_{3}\right) \geq 0$. Hence $\boldsymbol{\alpha}$ is in the domain of integrability of $K_{\boldsymbol{\alpha}}$.

The corollary covers all interesting cases for spherical unitary series, provided one excludes the trivial representation. In fact, the parameter $\lambda$ for such a representation can be chosen either as pure imaginary (principal series) or satisfying $0<\lambda<\rho$ (complementary series, excluding the trivial representation). 


\subsection{Analytic continuation of $\mathcal{K}_{\alpha}$}

The main result of this section concerns the analytic continuation of $\mathcal{K}_{\boldsymbol{\alpha}}$ beyond its domain of integrability.

Theorem 2.3. The map $\boldsymbol{\alpha} \longmapsto \mathcal{K}_{\boldsymbol{\alpha}}$, originally defined for $\boldsymbol{\alpha}$ in in the domain described by the conditions (24) and (25), valued in $\mathcal{D}^{\prime}(S \times S \times S)$ can be extended meromorphically with at most simple poles along the family of hyperplanes in $\mathbb{C} \times \mathbb{C} \times \mathbb{C}$ defined by the equations

$$
\begin{gathered}
\alpha_{j}=-\rho-2 k, \quad j=1,2,3, \quad k \in \mathbb{N} \\
\alpha_{1}+\alpha_{2}+\alpha_{3}=-\rho-2 k, \quad k \in \mathbb{N}
\end{gathered}
$$

Proof. Let $f$ be in $\mathcal{C}^{\infty}(S \times S \times S)$ and consider the integral

$$
\int_{S \times S \times S} K_{\boldsymbol{\alpha}}\left(x_{1}, x_{2}, x_{3}\right) f\left(x_{1}, x_{2}, x_{3}\right) d \sigma\left(x_{1}\right) d \sigma\left(x_{2}\right) d \sigma\left(x_{3}\right) .
$$

to be meromorphically continued. Repeating the argument given supra during the discussion of the integrability of the kernel $K_{\boldsymbol{\alpha}}$, we may assume that $f$ has its support contained in a small neigborhood of the point $\left(\mathbf{1}^{+}, \mathbf{1}^{+}, \mathbf{1}^{+}\right)$. Further, transfer the integral to the noncompact picture (cf (23) $)$, and study the analytic continuation of

$$
\boldsymbol{\alpha} \longmapsto \mathcal{J}_{\boldsymbol{\alpha}}\left((f \circ c) \Psi_{\boldsymbol{\alpha}}\right)
$$

Now $\varphi=f \circ c$ is in $\mathcal{C}_{c}^{\infty}\left(\mathbb{R}^{n-1} \times \mathbb{R}^{n-1} \times \mathbb{R}^{n-1}\right)$, and both $\boldsymbol{\alpha} \longmapsto \Psi_{\boldsymbol{\alpha}}$ and $\boldsymbol{\alpha} \longmapsto \Psi_{\boldsymbol{\alpha}}{ }^{-1}$ are entire fonctions on $\mathbb{C}^{3}$, so that it is equivalent to study the meromorphic continuation of $\mathcal{J}_{\boldsymbol{\alpha}}$ as a distribution on $\mathbb{R}^{n-1} \times \mathbb{R}^{n-1} \times \mathbb{R}^{n-1}$.

The kernel $J_{\alpha}$ is invariant by translations by "diagonal vectors". To take advantage of this remark, make the change of variables

$$
z_{1}=y_{1}, \quad z_{2}=y_{1}-y_{3}, \quad z_{3}=y_{1}-y_{2}
$$

in the integral

$$
\mathcal{J}_{\boldsymbol{\alpha}}(\varphi)=\int\left|y_{1}-y_{2}\right|^{-\rho+\alpha_{3}}\left|y_{2}-y_{3}\right|^{-\rho+\alpha_{1}}\left|y_{3}-y_{1}\right|^{-\rho+\alpha_{2}} \varphi\left(y_{1}, y_{2}, y_{3}\right) d y_{1} d y_{2} d y_{3}
$$

to obtain

$$
\mathcal{J}_{\boldsymbol{\alpha}}(\varphi)=\int_{\mathbb{R}^{n-1} \times \mathbb{R}^{n-1}}\left|z_{2}\right|^{-\rho+\alpha_{2}}\left|z_{3}\right|^{-\rho+\alpha_{3}}\left|z_{2}-z_{3}\right|^{-\rho+\alpha_{1}} \psi\left(z_{2}, z_{3}\right) d z_{2} d z_{3}
$$


where we have set

$$
\psi\left(z_{2}, z_{3}\right)=\int_{\mathbb{R}^{n-1}} \varphi\left(z_{1}, z_{1}-z_{3}, z_{1}-z_{2}\right) d z_{1} .
$$

Now observe that $\psi$ is in $\mathcal{C}_{c}^{\infty}\left(\mathbb{R}^{n-1} \times \mathbb{R}^{n-1}\right)$. Hence we are reduced to studying the analytic continuation of the distribution $\mathcal{I}_{\boldsymbol{\alpha}}$ on $\mathbb{R}^{n-1} \times \mathbb{R}^{n-1}$ defined by

$$
\mathcal{I}_{\boldsymbol{\alpha}}(\psi)=\int_{\mathbb{R}^{n-1} \times \mathbb{R}^{n-1}} I_{\boldsymbol{\alpha}}\left(z_{2}, z_{3}\right) \psi\left(z_{2}, z_{3}\right) d z_{2} d z_{3}
$$

for $\psi$ in $\mathbb{C}_{c}^{\infty}\left(\mathbb{R}^{n-1} \times \mathbb{R}^{n-1}\right)$, where we set

$$
I_{\boldsymbol{\alpha}}\left(z_{2}, z_{3}\right)=\left|z_{2}\right|^{-\rho+\alpha_{2}}\left|z_{3}\right|^{-\rho+\alpha_{3}}\left|z_{2}-z_{3}\right|^{-\rho+\alpha_{1}} .
$$

For $\delta>0$, consider the following open subsets of $\mathbb{R}^{n-1} \times \mathbb{R}^{n-1}$

$$
\begin{gathered}
\mathcal{U}_{0}=\left\{\left(z_{2}, z_{3}\right),\left|z_{2}\right|<\delta,\left|z_{3}\right|<\delta,\left|z_{2}-z_{3}\right|<\delta\right\} \\
\mathcal{U}_{1}=\left\{\left(z_{2}, z_{3}\right),\left|z_{2}\right|>\frac{\delta}{2},\left|z_{3}\right|>\frac{\delta}{2},\left|z_{2}-z_{3}\right|<\frac{\delta}{2}\right\} \\
\mathcal{U}_{2}=\left\{\left(z_{2}, z_{3}\right),\left|z_{2}\right|<\frac{\delta}{2},\left|z_{3}\right|>\frac{\delta}{2},\left|z_{3}-z_{2}\right|>\frac{\delta}{2}\right\} \\
\mathcal{U}_{3}=\left\{\left(z_{2}, z_{3}\right),\left|z_{2}\right|>\frac{\delta}{2},\left|z_{3}\right|<\frac{\delta}{2},\left|z_{2}-z_{3}\right|>\frac{\delta}{2}\right\} \\
\mathcal{U}_{\infty}=\left\{\left(z_{2}, z_{3}\right),\left|z_{2}\right|>\frac{\delta}{2},\left|z_{3}\right|>\frac{\delta}{2},\left|z_{2}-z_{3}\right|>\frac{\delta}{2}\right\} .
\end{gathered}
$$

The family of these five open sets form a covering of $\mathbb{R}^{n-1} \times \mathbb{R}^{n-1}$. Let study the restriction of the distribution $\mathcal{I}_{\boldsymbol{\alpha}}$ to each of these five open subsets.

If $\operatorname{Supp}(\psi) \subset \mathcal{U}_{\infty}$, there the $\mathcal{I}_{\boldsymbol{\alpha}}(\psi)$ extends as an entire function, because $I_{\boldsymbol{\alpha}}$ has no singularity on $\mathcal{U}_{\infty}$. Next assume that $\operatorname{Supp}(\psi) \subset \mathcal{U}_{2}$. Set

$$
\phi_{\boldsymbol{\alpha}}\left(z_{2}\right)=\int_{\mathbb{R}^{n-1}}\left|z_{3}\right|^{-\rho+\alpha_{3}}\left|z_{2}-z_{3}\right|^{-\rho+\alpha_{1}} \psi\left(z_{2}, z_{3}\right) d z_{3}
$$

so that

$$
\mathcal{I}_{\boldsymbol{\alpha}}(\psi)=\int_{\mathbb{R}^{n-1}}\left|z_{2}\right|^{-\rho+\alpha_{2}} \phi_{\boldsymbol{\alpha}}\left(z_{2}\right) d z_{2} .
$$

As $\left|z_{3}\right|$ and $\left|z_{2}-z_{3}\right|$ are bounded from below on $\mathcal{U}_{2}$, the function $\phi_{\boldsymbol{\alpha}}$ is in $\mathcal{C}_{c}^{\infty}\left(\mathbb{R}^{n-1}\right)$ and $\boldsymbol{\alpha} \longmapsto \phi_{\boldsymbol{\alpha}}$ is an entire function on $\mathbb{C}^{3}$. On $\mathbb{R}^{n-1}$, the distribution-valued function $s \longmapsto\left|z_{2}\right|^{s}$ extends meromorphically on $\mathbb{C}$, with 
simple poles at $s=-(n-1)-2 k, k \in \mathbb{N}$ (see [6]), so that the integral (30) extends meromorphically to $\mathbb{C}^{3}$ with simple poles along the hyperplanes $\alpha_{2}=-\rho-2 k$. A similar analysis can be done over $\mathcal{U}_{1}$ and $\mathcal{U}_{3}$.

To sum up what we have already proved, introduce the family $\mathcal{M}$ of meromorphic functions on $\mathbb{C}^{3}$ having at most simple poles along the hyperplanes $\left\{\alpha_{j}=-\rho-2 k\right\}, j=1,2,3, k \in \mathbb{N}$. Notice that they are the hyperplanes corresponding to the conditions (26) .

Proposition 2.2. Let $\psi$ be in $\mathcal{C}_{c}^{\infty}\left(\mathbb{R}^{n-1} \times \mathbb{R}^{n-1}\right)$ and assume that $\operatorname{Supp}(\psi) \not \supset$ $(0,0)$. Then the function $\boldsymbol{\alpha} \mapsto \mathcal{I}_{\boldsymbol{\alpha}}(\psi)$ belongs to the class $\mathcal{M}$.

Proof. In fact, choose $\delta$ small enough so that $\operatorname{Supp}(\psi) \cap \mathcal{U}_{0}=\emptyset$. Use a partition of unity to write $\psi$ as

$$
\psi=\psi_{1}+\psi_{2}+\psi_{3}+\psi_{\infty}
$$

where $\psi_{j} \in \mathcal{C}_{c}^{\infty}\left(\mathbb{R}^{n-1} \times \mathbb{R}^{n-1}\right)$ and $\operatorname{Supp}\left(\psi_{j}\right) \subset \mathcal{U}_{j}, j=1,2,3, \infty$. The previous analysis shows that $\boldsymbol{\alpha} \longmapsto \mathcal{I}_{\boldsymbol{\alpha}}\left(\psi_{j}\right)$ extends meromorphically on $\mathbb{C}^{3}$ with at most simple poles along the hyperplanes $\alpha_{j}=-\rho-2 k, k \in \mathbb{N}$ for $j=1,2,3$, whereas $\mathcal{I}_{\boldsymbol{\alpha}}\left(\psi_{\infty}\right)$ is an entire function of $\boldsymbol{\alpha}$.

Now we use a priori the existence of the meromorphic continuation of such integrals (see [13]). Moreover, the poles are located on a locally finite family of affine hyperplanes (of a rather specific type, but we won't need this result). Let $\mathcal{H}$ be such an hyperplane (to be determined), but not included in the family of hyperplanes given by conditions (26) . Let $\boldsymbol{\alpha}^{0}=\left(\alpha_{1}^{0}, \alpha_{2}^{0}, \alpha_{3}^{0}\right)$ be a regular point in $\mathcal{H}$, (i.e. not contained in any other hyperplane of poles). The Laurent coefficients at $\boldsymbol{\alpha}^{0}$ are distributions on $\mathbb{R}^{n-1} \times \mathbb{R}^{n-1}$, and, by Lemma 2.2, their supports have to be contained in $\{0,0\}$, hence they are derivatives of the Dirac measure $\delta_{(0,0)}$. So, if $\mathcal{I}_{\boldsymbol{\alpha}}$ does have a pole at $\boldsymbol{\alpha}^{0}$, there exists a smooth function $\rho$ on $\mathbb{R}^{n-1} \times \mathbb{R}^{n-1}$ with compact support and identically equal to 1 in a neigbourhood of $(0,0)$, and a polynomial $P$ on $\mathbb{R}^{n-1} \times \mathbb{R}^{n-1}$, homogeneous of degree $k$ such that $\mathcal{I}_{\boldsymbol{\alpha}}(\rho P)$ does not extend holomorphically at $\boldsymbol{\alpha}^{0}$. For $t \in \mathbb{R}^{*}$, let $\rho_{t}\left(z_{2}, z_{3}\right)=\rho\left(t z_{2}, t z_{3}\right)$. Use the change of variables $\left(z_{2} \mapsto t z_{2}, z_{3} \mapsto t z_{3}\right)$ to get 2 , for $\alpha \neq \alpha_{0}$

$$
\begin{gathered}
\mathcal{I}_{\boldsymbol{\alpha}}\left(\rho_{t} P\right)=\int_{\mathbb{R}^{n-1} \times \mathbb{R}^{n-1}}\left|z_{2}\right|^{-\rho+\alpha_{3}}\left|z_{3}\right|^{-\rho+\alpha_{2}}\left|z_{2}-z_{3}\right|^{-\rho+\alpha_{1}} \rho\left(t z_{2}, t z_{3}\right) P\left(z_{2}, z_{3}\right) d z_{2} d z_{3} \\
=|t|^{-\alpha_{3}-\alpha_{2}-\alpha_{1}-\rho} t^{-k} \mathcal{I}_{\boldsymbol{\alpha}}(\rho P) .
\end{gathered}
$$

\footnotetext{
${ }^{2}$ Following a traditional way, we write $\mathcal{I}_{\boldsymbol{\alpha}}(\varphi)$ as an integral. What is really used here is merely the homogeneity of $\mathcal{I}_{\boldsymbol{\alpha}}$.
} 
Hence

$$
\left(1-|t|^{-\alpha_{3}-\alpha_{2}-\alpha_{1}-\rho t^{-k}}\right) \mathcal{I}_{\boldsymbol{\alpha}}(\rho P)=\mathcal{I}_{\boldsymbol{\alpha}}\left(\left(\rho-\rho_{t}\right) P\right) .
$$

Now observe that the support of $\left(\rho-\rho_{t}\right) P$ does not contain $(0,0)$, and hence, $\boldsymbol{\alpha} \longmapsto \mathcal{I}_{\boldsymbol{\alpha}}\left(\left(\rho-\rho_{t}\right) P\right)$ belongs to $\mathcal{M}$. The assumption that $\mathcal{I}_{\boldsymbol{\alpha}}(\rho P)$ does have a pole at $\boldsymbol{\alpha}_{0}$ forces the condition

$$
\forall t \in \mathbb{R}^{*} \quad 1-|t|^{-\alpha_{3}^{0}-\alpha_{2}^{0}-\alpha_{1}^{0}-\rho t^{-k}}=0 .
$$

In turn, this condition amounts to

$$
k \in 2 \mathbb{N}, \quad \alpha_{1}^{0}+\alpha_{2}^{0}+\alpha_{3}^{0}=-\rho-k .
$$

Moreover, (31) shows that $\mathcal{I}_{\boldsymbol{\alpha}}$ has at most a simple pole along the corresponding hyperplane. This achieves the proof of Theorem 2.3 .

As the invariance condition remains true by analytic continuation, Theorem 2.3 can be reformulated for trilinear invariant functionals (cf Theorem 2.1).

Theorem 2.4. Let $\boldsymbol{\lambda}=\left(\lambda_{1}, \lambda_{2}, \lambda_{3}\right)$ in $\mathbb{C}^{3}$. Assume that

$$
\begin{array}{r}
-\lambda_{1}+\lambda_{2}+\lambda_{3} \notin-\rho-2 \mathbb{N} \\
\lambda_{1}-\lambda_{2}+\lambda_{3} \notin-\rho-2 \mathbb{N} \\
\lambda_{1}+\lambda_{2}-\lambda_{3} \notin-\rho-2 \mathbb{N} \\
\lambda_{1}+\lambda_{2}+\lambda_{3} \notin-\rho-2 \mathbb{N}
\end{array}
$$

Set $\boldsymbol{\alpha}=\left(\alpha_{1}, \alpha_{2}, \alpha_{3}\right)$ where (cf (19))

$$
\begin{aligned}
& \alpha_{1}=-\lambda_{1}+\lambda_{2}+\lambda_{3} \\
& \alpha_{2}=-\lambda_{2}+\lambda_{3}+\lambda_{1} \\
& \alpha_{3}=-\lambda_{3}+\lambda_{1}+\lambda_{2} .
\end{aligned}
$$

Then $\left(f_{1}, f_{2}, f_{3}\right) \longmapsto \mathcal{T}_{\boldsymbol{\lambda}}\left(f_{1}, f_{2}, f_{3}\right)=\mathcal{K}_{\boldsymbol{\alpha}}\left(f_{1} \otimes f_{2} \otimes f_{3}\right)$ is a well defined non trivial trilinear invariant functional w.r.t. the representations $\left(\pi_{\lambda_{1}}, \pi_{\lambda_{2}}, \pi_{\lambda_{3}}\right)$.

The next result was obtained some time ago by the present authors, and has been generalized to other geometric situations in a collaboration with T. Kobayashi and M. Pevzner (see [3]). To state the result, consider the evaluation of the functional $\mathcal{K}_{\boldsymbol{\alpha}}$ against the function $\mathbb{I}_{S} \otimes \mathbb{I}_{S} \otimes \mathbb{I}_{S}$, where $\mathbb{I}_{S}$ is the function which is identically 1 on $S$. Let 
$I(\boldsymbol{\lambda})=K(\boldsymbol{\alpha})=\int_{S} \int_{S} \int_{S} k_{\alpha_{1}}\left(x_{2}, x_{3}\right) k_{\alpha_{2}}\left(x_{3}, x_{1}\right) k_{\alpha_{3}}\left(x_{1}, x_{2}\right) d \sigma\left(x_{1}\right) d \sigma\left(x_{2}\right) d \sigma\left(x_{3}\right)$

where $\boldsymbol{\alpha}$ and $\boldsymbol{\lambda}$ are related by the relations (19).

Proposition 2.3. Let $\boldsymbol{\lambda}=\left(\lambda_{1}, \lambda_{2}, \lambda_{3}\right)$ in $\mathbb{C}^{3}$ and assume that the conditions (32) are satisfied. Then

$$
\begin{gathered}
I(\boldsymbol{\lambda})=\left(\frac{\sqrt{\pi}}{2}\right)^{3(n-1)} 2^{\lambda_{1}+\lambda_{2}+\lambda_{3}} \ldots \\
\ldots \frac{\Gamma\left(\frac{\lambda_{1}+\lambda_{2}+\lambda_{3}+\rho}{2}\right) \Gamma\left(\frac{-\lambda_{1}+\lambda_{2}+\lambda_{3}+\rho}{2}\right) \Gamma\left(\frac{\lambda_{1}-\lambda_{2}+\lambda_{3}+\rho}{2}\right) \Gamma\left(\frac{\lambda_{1}+\lambda_{2}-\lambda_{3}+\rho}{2}\right)}{\Gamma\left(\rho+\lambda_{1}\right) \Gamma\left(\rho+\lambda_{2}\right) \Gamma\left(\rho+\lambda_{3}\right)}
\end{gathered}
$$

Remark Both sides of the formula are meromorphic functions on $\mathbb{C}^{3}$, and they are equal where defined. Notice that $I(\boldsymbol{\lambda})=K(\boldsymbol{\alpha})$ has simple poles exactly as prescribed by Theorem 2.3 .

This result allows to strengthen the previous theorem. Define

$$
\widetilde{\mathcal{K}}_{\alpha}=\frac{\mathcal{K}_{\alpha}}{\Gamma\left(\frac{\lambda_{1}+\lambda_{2}+\lambda_{3}+\rho}{2}\right) \Gamma\left(\frac{-\lambda_{1}+\lambda_{2}+\lambda_{3}+\rho}{2}\right) \Gamma\left(\frac{\lambda_{1}-\lambda_{2}+\lambda_{3}+\rho}{2}\right) \Gamma\left(\frac{\lambda_{1}+\lambda_{2}-\lambda_{3}+\rho}{2}\right)}
$$

Theorem 2.5. The distribution-valued function $\boldsymbol{\alpha} \mapsto \widetilde{\mathcal{K}}_{\boldsymbol{\alpha}}$ extends as an entire holomorphic function on $\mathbb{C}^{3}$. The trilinear functional $\tilde{\mathcal{T}}_{\boldsymbol{\lambda}}$ defined by

$$
\widetilde{\mathcal{T}}_{\boldsymbol{\lambda}}\left(f_{1}, f_{2}, f_{3}\right)=\widetilde{\mathcal{K}}_{\boldsymbol{\alpha}}\left(f_{1} \otimes f_{2} \otimes f_{3}\right)
$$

on $\left.\mathcal{C}^{\infty}(S) \times \mathcal{C}^{\infty}(S) \times \mathcal{C}^{\infty}(S)\right)$ is invariant with respect to $\left(\pi_{\lambda_{1}}, \pi_{\lambda_{2}}, \pi_{\lambda_{3}}\right)$, where $\left(\lambda_{1}, \lambda_{2}, \lambda_{3}\right)$ are related to $\left(\alpha_{1}, \alpha_{2}, \alpha_{3}\right)$ by the relations (19). The trilinear form $\widetilde{\mathcal{T}}_{\boldsymbol{\lambda}}$ is not identically 0 provided the two following conditions are not simultaneously realized

- $\exists j, 1 \leq j \leq 3, \quad \lambda_{j} \in-\rho-\mathbb{N}$

- (at least) one of the conditions (32) is satisfied.

Proof. The function $\boldsymbol{\alpha} \longmapsto \widetilde{\mathcal{K}}_{\boldsymbol{\alpha}}$ extends holomorphically near any regular point of the hyperplanes defined by conditions (26) and (27), so is holmomorphic outside of the set where ar least two hyperplanes of poles meet. But this set is of codimension 2 and hence $\boldsymbol{\alpha} \longmapsto \widetilde{\mathcal{K}}_{\boldsymbol{\alpha}}$ extends as a holomorphic function to all of $\mathbb{C}^{3}$. If none of the conditions (32) is satisfied, then $\widetilde{\mathcal{K}}_{\boldsymbol{\alpha}}$ is a multiple $(\neq 0)$ of $\mathcal{K}_{\boldsymbol{\alpha}}$ which is different from 0 on $\Omega$. If $\lambda_{j} \notin-\rho+\mathbb{N}$ for $j=1,2,3$, then $I(\boldsymbol{\lambda}) \neq 0$ and hence $\mathcal{K}_{\boldsymbol{\alpha}}$ is not identically 0 . 


\section{Uniqueness of the invariant trilinear form}

\subsection{Induced representations and line bundles}

For this part, it is useful to realize the representation $\pi_{\lambda}$ as acting on smooth sections of a line bundle over $S$.

Recall that the stabilizer of $\mathbf{1}^{+}$in $G$ is the parabolic subgroup $P=$ $M A N$. The left invariant Haar measure on $P$ is

$$
\int_{P} f(p) d p=\int_{M} \int_{A} \int_{N} f(\operatorname{man}) d m d a d n
$$

For $\lambda \in \mathbb{C}$, denot by $\chi_{\lambda}$ the character of $P$ defined by

$$
\chi_{\lambda}\left(m a_{t} n\right)=e^{t \lambda} .
$$

The modular function of $P$ is given by $\delta_{P}(\operatorname{man})=e^{-2 \rho(\log a)}=\chi_{-2 \rho}(p)$ (see e.g. [17] Lemma 5.5.1.1), so that, for any $q$ in $P$

$$
\int_{P} f(p q) d p=\chi_{2 \rho}(q) \int_{P} f(p) d p
$$

Let $\mathcal{E}_{\lambda}$ be the space of functions $f$ in $\mathcal{C}^{\infty}(G)$ which, for all $g$ in $G, p$ in $P$ satisfy

$$
f(g p)=\chi_{-(\rho+\lambda)}(p) f(g)
$$

Then $G$ acts on $\mathcal{E}_{\lambda}$ by

$$
\Pi_{\lambda}(g) f(\gamma)=f\left(g^{-1} \gamma\right)
$$

To any function $f$ in $\mathcal{E}_{\lambda}$, associate the function $\tilde{f}$ defined on $S$ by the formula

$$
\tilde{f}(s)=f(k)
$$

where $k$ is any element in $K$ satisfying $k \mathbf{1}^{+}=s$. As $f(k m)=f(m)$ for any $m$ in $M$, the right handside of (38) does not depend on $m$, but merely on $k \mathbf{1}^{+}=s$.

Now, let $g$ be in $G$, let $s$ be in $S$, and let $k$ be in $K$ such that $k \mathbf{1}^{+}=s \in S$. Let

$$
g^{-1} k=k\left(g^{-1} k\right) a\left(g^{-1} k\right) n
$$

be the Iwasawa decomposition of $g^{-1} k$. Then

$$
k\left(g^{-1} k\right) \mathbf{1}^{+}=\left(g^{-1} k\right)\left(\mathbf{1}^{+}\right)=g^{-1}(s)
$$

and

$$
\kappa\left(g^{-1}, s\right)=\kappa\left(g^{-1} k, \mathbf{1}^{+}\right)=\chi_{-1}\left(a\left(g^{-1} k\right)\right)
$$


Hence,

$$
\left(\Pi_{\lambda}(g) f\right)^{\sim}(s)=f\left(g^{-1} k\right)=f\left(k\left(g^{-1} k\right) a\left(g^{-1} k\right) n\right)=\kappa\left(g^{-1}, s\right)^{\rho+\lambda} \tilde{f}\left(g^{-1}(s)\right)
$$

so that $f \mapsto f^{\sim}$ is an intertwining operator for $\Pi_{\lambda}$ and $\pi_{\lambda}$.

Let $\chi$ be a character of $A$. Denote by $\mathbb{C}_{\chi}$ the representation of $P$ on $\mathbb{C}$ given by

$$
\operatorname{man} . z=\chi(a) z .
$$

Form the line bundle $L=L_{\chi}=G \times{ }_{P} \mathbb{C}_{\chi}$ over $S$, and let $\mathcal{L}=\mathcal{L}_{\chi}^{\infty}$ be the space of $\mathcal{C}^{\infty}$ sections of $L$. Then $G$ acts naturally on $\mathcal{L}$ by left translations. As sections of $L$ can be identified with functions on $G$ transforming by $\chi^{-1}$ under the right action of $P$, the representation of $G$ on $\mathcal{L}$ is equivalent to $\pi_{\lambda}$ if $\chi=\chi_{\rho+\lambda}$.

Now take three characters $\chi_{1}=\chi_{\rho+\lambda_{1}}, \chi_{2}=\chi_{\rho+\lambda_{2}}, \chi_{3}=\chi_{\rho+\lambda_{3}}$ of $A$, set $\boldsymbol{\lambda}=\left(\lambda_{1}, \lambda_{2}, \lambda_{3}\right)$ and form the "exterior" product bundle $L_{\boldsymbol{\lambda}}=L_{\chi_{1}} \otimes L_{\chi_{2}} \otimes$ $L_{\chi_{3}}$ as a line bundle over $S \times S \times S$. Let $\mathcal{L}_{\boldsymbol{\lambda}}$ be the space of $\mathcal{C}^{\infty}$ sections of this bundle. Let the group $G$ acts naturally on this space by diagonal action. Then a trilinear invariant functional on $\mathcal{C}^{\infty}(S) \times \mathcal{C}^{\infty}(S) \times \mathcal{C}^{\infty}(S)$ for $\pi_{\lambda_{1}}, \pi_{\lambda_{2}}, \pi_{\lambda_{3}}$ corresponds to an invariant linear form on $\mathcal{L}_{\lambda}$.

In turn, such an invariant linear functional on $\mathcal{L}_{\boldsymbol{\lambda}}$ can be viewed as an invariant distribution density for the dual bundle $L_{\boldsymbol{\lambda}}^{*}$ (see Appendix).

The main tool to study these invariant distributions is Bruhat's theory, which is presented in the Appendix. We use heavily the description of orbits of $G$ in $S \times S \times S$ (cf Proposition 1.2).

Theorem 3.1. Let $\boldsymbol{\lambda}=\left(\lambda_{1}, \lambda_{2}, \lambda_{3}\right)$ be three complex numbers, let $\boldsymbol{\alpha}=$ $\left(\alpha_{1}, \alpha_{2}, \alpha_{3}\right)$ be defined as in (19) and assume that they satisfy the following generic conditions

i) $\alpha_{j} \notin-\rho-2 \mathbb{N}$ for $j=1,2,3$

ii) $\alpha_{1}+\alpha_{2}+\alpha_{3} \notin-\rho-2 \mathbb{N}$.

Then any trilinear invariant form for $\pi_{\lambda_{1}}, \pi_{\lambda_{2}}, \pi_{\lambda_{3}}$ is proportional to the form $\mathcal{T}_{\boldsymbol{\lambda}}$.

Proof. Denote by $T=T_{\boldsymbol{\lambda}}$ the distribution density on $S \times S \times S$ for the bundle $L_{\lambda}^{*}$ corresponding to an invariant trilinear form for $\pi_{\lambda_{1}}, \pi_{\lambda_{2}}, \pi_{\lambda_{3}}$.

\section{Step 1 : contribution of $\mathcal{O}_{0}$}

We use freely of the notation presented in the appendix. Consider the restriction $T^{\prime}$ of $T$ to the open orbit $\mathcal{O}_{0}$. Then $\mathcal{O}_{0}$ is a homogeneous space under $G$, the stabilizer $H$ of the base point $\left(\mathbf{1}^{+}, \mathbf{1}^{-}, e_{2}\right)$ is compact. There is 
an invariant measure on $G / H$, the group $H$ acts trivially on the fiber of $L_{\boldsymbol{\lambda}}^{*}$, so there exists exactly one (up to constant) invariant distribution given by a smooth density. But we already know that $K_{\boldsymbol{\alpha}}$ has the right transformation property. Hence on $\mathcal{O}_{0}$, after multiplication by a constant, we may assume that $T^{\prime}$ coincides with the restriction of $\mathcal{K}_{\boldsymbol{\alpha}}$ to $\mathcal{O}_{0}$. But the assumptions on $\boldsymbol{\lambda}$ guarantee that $\mathcal{K}_{\boldsymbol{\alpha}}$ can be extended (by analytic continuation) as an invariant distribution on $S \times S \times S$. Hence, in order to prove that $T$ is a multiple of $\mathcal{K}_{\boldsymbol{\alpha}}$, (i.e. to prove the uniqueness statement) we need only to prove that an invariant distribution which vanishes on $\mathcal{O}_{0}$ is identically 0 . In other words, we may (and hence do) assume that

$$
\operatorname{Supp}(T) \subset \cup_{1 \leq j \leq 4} \mathcal{O}_{j} .
$$

and proceed further to prove that $T=0$.

Let us mention that the argument given here should be modified for the case of the circle (i.e. as $n=2$ ), because there are two orbits for $S O_{0}(1,2)$ in $S \times S \times S$. To restore uniqueness, one can also consider the full group $O(1,2)$ instead. The rest of the proof is unchanged.

\section{Step 2 : contribution of $\mathcal{O}_{1}, \mathcal{O}_{2}, \mathcal{O}_{3}$}

We now show that

$$
\operatorname{Supp}(T) \cap \mathcal{O}_{3}=\emptyset .
$$

Observe that for $1 \leq j \leq 3$

$$
\overline{\mathcal{O}_{j}}=\mathcal{O}_{j} \cup \mathcal{O}_{4}
$$

so that $\left(\mathcal{O}_{1} \cup \mathcal{O}_{2} \cup \mathcal{O}_{4}\right)$ is a closed subset of $S \times S \times S$. Let

$$
X=S \times S \times S \backslash\left(\mathcal{O}_{1} \cup \mathcal{O}_{2} \cup \mathcal{O}_{4}\right) .
$$

Then $X$ is an open submanifold of $S \times S \times S$, acted by $G$, and $\mathcal{O}_{3}$ is a closed orbit of $G$ in $X$. Let $T^{\prime \prime}$ be the restriction of $T$ to $X$, so that $S u p p\left(T^{\prime \prime}\right) \subset \mathcal{O}_{3}$. Now apply Bruhat's theory.

The normal space at the base point $\left(\mathbf{1}^{+}, \mathbf{1}^{+}, \mathbf{1}^{-}\right)$is identified (via the Riemannian metric on $S \times S \times S$ ) with

$$
N_{o}=\left\{(\xi,-\xi, 0), \xi \in T_{1} S\right\} \simeq \mathbb{R}^{n-1}
$$

The stabilizer of of the base point is $H=A M$, and there is an invariant measure on $\mathcal{O}_{3}$, so that $\chi_{0} \equiv 1$. The group $M$ acts on $N_{0}$ by its natural action. As $M$ modules, $N_{0}$ and its dual are equivalent, so that the spaces 
$S^{k}\left(N_{0}\right)$ and $\mathcal{P}_{k}\left(N_{0}\right)$ are equivalent $M$-modules. The space of $M$-invariants in $\mathcal{P}_{k}\left(N_{0}\right)$ is 0 if $k$ is odd (and one-dimensional, generated by $|\xi|^{k}$ if $k$ is even, but we won't need this fact). On the other hand, an element $a_{t}$ of A acts on $N_{0}$ by dilation by $e^{-t}$, so that it acts on $S^{k}\left(N_{0}\right)$ by multiplication by $e^{-k t}$. The element $a_{t}$ acts on the fiber $L_{0}$ of $L_{\chi_{1}, \chi_{2}, \chi_{3}}$ at $\left(\mathbf{1}^{+}, \mathbf{1}^{+}, \mathbf{1}^{-}\right)$by $e^{\left(\rho+\lambda_{1}+\rho+\lambda_{2}-\rho-\lambda_{3}\right) t}$. Hence the element $a_{t}$ acts on $S^{k}\left(N_{0}\right) \otimes L_{0}^{*}$ by multiplication by

$$
e^{\left(-k-\rho-\left(\lambda_{1}+\lambda_{2}-\lambda_{3}\right)\right) t}
$$

The assumptions on $\boldsymbol{\lambda}$ (more precisely $\alpha_{3}=\lambda_{1}+\lambda_{2}-\lambda_{3} \notin-\rho-2 \mathbb{N}$ ) guarantee that

$$
\left(\mathcal{S}_{k}\left(N_{0}\right) \otimes L_{0}^{*} \otimes \mathbb{C}_{\chi_{0}^{-1}}\right)^{H}=0
$$

for any $k \in \mathbb{N}$. Hence, by Corollary $\mathrm{A} \mathbb{1}$, there is no non-trivial invariant distribution supported in $\mathcal{O}_{3}$.

Repeating the argument for $\mathcal{O}_{2}$ and $\mathcal{O}_{1}$, we may (and hence do) assume now that

$$
\operatorname{Supp}(T) \subset \mathcal{O}_{4}
$$

\section{Third step : contribution of $\mathcal{O}_{4}$}

Here we take $X=S \times S \times S$, as $\mathcal{O}_{4}$ is closed. The stabilizer of the base-point $\left(\mathbf{1}^{+}, \mathbf{1}^{+}, \mathbf{1}^{+}\right)$is $P=M A N$. The character $\chi_{0}$ is given by

$$
\chi_{0}\left(m a_{t} n\right)=e^{-2 \rho t} \text {. }
$$

The normal space at $\left(\mathbf{1}^{+}, \mathbf{1}^{+}, \mathbf{1}^{+}\right)$can be identified with

$$
N_{0}=\left\{\left(\xi_{1}, \xi_{2}, \xi_{3}\right), \xi_{j} \in T_{1} S, \xi_{1}+\xi_{2}+\xi_{3}=0\right\} \simeq \mathbb{R}^{n-1} \oplus \mathbb{R}^{n-1} .
$$

The group $M$ acts on $N_{0}$ by its natural action on each factor. Again, as $M$ modules, the space $S^{k}\left(N_{0}\right)$ is isomorphic to $\mathcal{P}_{k}\left(N_{0}\right)$. The algebra of $S O(n-1)$ invariant polynomials on $\mathbb{R}^{n-1} \oplus \mathbb{R}^{n-1}$ is generated (as an algebra) by $|\xi|^{2},|\eta|^{2},\langle\xi, \eta\rangle$. Hence $S^{k}\left(N_{0}\right)^{M}=\{0\}$ if $k$ is odd. On the other hand, an element $a_{t}$ of $A$ acts on $N_{0}$ by multiplication by $e^{-t}$, hence on $S_{k}\left(N_{0}\right)$ by multiplication by $e^{-k t}$. It acts on the fiber $L_{0}$ by $e^{\left(\rho+\lambda_{1}+\rho+\lambda_{2}+\rho+\lambda_{3}\right) t}$. Hence $a_{t}$ acts on $S^{k}\left(N_{0}\right) \otimes L_{0}^{*} \otimes \mathbb{C}_{\chi_{o}^{-1}}$ by

$$
e^{\left(-k-\rho-\left(\lambda_{1} \lambda_{2}+\lambda_{3}\right)\right) t}
$$

The assumptions on $\boldsymbol{\lambda}$ (namely $\lambda_{1}+\lambda_{2}+\lambda_{3} \notin-\rho-2 \mathbb{N}$ ) guarantee that

$$
\left(\mathcal{S}_{k}\left(N_{0}\right) \otimes L_{0}^{*} \otimes \mathbb{C}_{\chi_{0}^{-1}}\right)^{H}=\{0\}
$$

for any $k \in \mathbb{N}$. Hence, by Corollary $\mathrm{A} \mathbb{1}$, there is no non-trivial invariant distribution supported in $\mathcal{O}_{4}$. The uniqueness statement follows. 


\section{Another construction of a trilinear invariant form}

In this section we present a different construction of the invariant trilinear form $\mathcal{T}_{\boldsymbol{\lambda}}$. Trilinear forms are connected with tensor products of representations. Roughly speaking, a trilinear form on $\mathcal{H}_{1} \times \mathcal{H}_{2} \times \mathcal{H}_{3}$ can be realized as an invariant bilinear pairing between $\mathcal{H}_{1} \otimes \mathcal{H}_{2}$ and $\mathcal{H}_{3}$. Although this point of view breaks the (sort of) symmetry between the three factors, it produces interesting relations with questions about tensor products of representations (cf [11], which was the main source of inspiration for this section). Our presentation of the construction is formal and we don't work out the estimates and analytical aspects of the construction, which would follow along similar lines as in previous sections.

\subsection{The space $S_{\text {T }}^{2}$ as a symmetric space}

Recall that $S_{\top}^{2}=\{(x, y) \in S \times S, x \neq y\}$ is the orbit of $\left(\mathbf{1}^{+}, \mathbf{1}^{-}\right)$under $G$. The stabilizer in $G$ of $\left(\mathbf{1}^{+}, \mathbf{1}^{-}\right)$is the subgroup $H=M A$.

$$
\begin{aligned}
& \text { Let } J=\left(\begin{array}{ccccc}
-1 & 0 & 0 & \ldots & 0 \\
0 & -1 & 0 & \ldots & 0 \\
0 & 0 & 1 & & 0 \\
\vdots & \vdots & & \ddots & \vdots \\
0 & 0 & 0 & \ldots & 1
\end{array}\right) \text {. Then } J^{t}=J^{-1}=J \text {. The map } \\
& g \longmapsto J g J
\end{aligned}
$$

is an involutive automorphism of $G$. The set of fixed points of this involution is the subgroup $\widetilde{H}=H \sqcup H^{-}$,

$$
H^{-}=\left\{h=\left(\begin{array}{ccc}
\cosh t & -\sinh t & 0 \\
\sinh t & -\cosh t & 0 \\
0 & 0 & k
\end{array}\right), \quad t \in \mathbb{R}, k \in O(n-1), \operatorname{det} k=-1\right\} .
$$

So $H$ is the neutral component of the fixed points of an involutive automorphism of $G$. In other words, $S_{\top}^{2}$ can be realized as the symmetric space $G / H$ via

$$
G / H \ni g \longmapsto\left(g\left(\mathbf{1}^{+}\right), g\left(\mathbf{1}^{-}\right)\right) .
$$

There exists a unique (up to a positive real number) $G$-invariant measure on $S_{\top}^{2}$, namely

$$
\int_{G / H} f(x) d \mu(x)=\int_{S} \int_{S} f(s, t) \frac{d s d t}{|s-t|^{2(n-1)}} .
$$


Another description of $G / H$ is as follows. Let $\mathcal{X}$ be the set of all 2dimensional oriented subspaces $\Pi$ in $\mathbb{R}^{1, n}$ such that the restriction of $q$ to $\Pi$ is of signature $(1,1)$. The space $\mathcal{X}$ is an open set in the Grassmannian $G(2, n+1)$ of oriented 2 -dimensional subspaces in $\mathbb{R}^{1, n}$. The group $G$ operates transitively on $\mathcal{X}$. To the couple $(s, t)$ in $S_{\text {T }}^{2}$, associate the 2-dimensional space $\Pi(s, t)=\mathbb{R} \widetilde{s} \oplus \mathbb{R} \widetilde{t}$, with the orientation for which $(\widetilde{s}, \widetilde{t})$ is a direct basis.

Proposition 4.1. The mapping $\Pi$ is a diffeomorphism from $S_{\top}^{2}$ onto $\mathcal{X}$.

Proof. Let $s, t$ be in $S_{\text {个 }}^{2}$. Then $\Pi(s, t)$ cannot be totally isotropic for $q$, because the maximally isotropic subspaces are of dimension 1 . As $\Pi(s, t)$ contains two independant isotropic vectors $(\widetilde{s}$ and $\widetilde{t})$, the signature of $q$ on $\Pi(s, t)$ has to be $(1,1)$. Hence $\Pi(s, t)$ belongs to $\mathcal{X}$. On the other hand, let $\Pi$ be in $\mathcal{X}$. Then the isotropic cone in $\Pi$ decomposes as the union of two distinct isotropic lines, say $D \cup \Delta$. The line $D$ (resp. $\Delta$ ) intersects the hyperplane $\left\{x_{0}=1\right\}$ at a point $\widetilde{s}$ (resp. $\widetilde{t}$ ) with $s$ (resp. $t$ ) in $S$. Permuting $D$ and $\Delta$ if needed, we may assume that the basis $(\widetilde{s}, \widetilde{t})$ corresponds to the orientation of $\Pi$. Then clearly $\Pi(s, t)=\Pi$. Thus $\Pi$ is a $1-1$ correspondance. The fact that it is a diffeomorphism is standard and left to the reader.

\subsection{An equivariant realization of $\pi_{\sigma} \otimes \pi_{\tau}$}

The group $H$ is connected, isomorphic to $\mathbb{R} \times S O(n-1)$. For $\zeta$ any complex number, the function $\nu_{\zeta}$ on $H$ defined by

$$
\nu_{\zeta}\left(\begin{array}{ccc}
\cosh t & \sinh t & 0 \\
\sinh t & \cosh t & 0 \\
0 & 0 & k
\end{array}\right)=e^{t \zeta}
$$

$(t \in \mathbb{R}, k \in M)$ is a character of $H$.

Form the line bundle $L_{\zeta}=G \times_{H} \mathbb{C}_{\zeta}$ over $G / H$. A section of $L_{\zeta}$ can be viewed as a $\mathcal{C}^{\infty}$ function $F: G \longrightarrow \mathbb{C}$ which satisfies

$$
F(g h)=\nu_{\zeta}(h)^{-1} F(g) .
$$

Denote by $\mathcal{L}_{\zeta}$ the space of smooth sections of $L_{\zeta}$ over $G / H$. The natural action of $G$ acts on $\mathcal{L}_{\zeta}$ by left translation is denoted by $I_{\zeta}$ :

$$
\left(I_{\zeta}(\gamma) F\right)(g)=F\left(\gamma^{-1} g\right)
$$

When $\zeta$ is pure imaginary, the character $\nu_{\zeta}$ is unitary. If $F$ is in $\mathcal{L}_{\zeta}$, then $|F(g h)|=|F(g)|$ for any $h$ in $H$. Hence the expression

$$
\|F\|^{2}=\int_{G / H}|F(g)|^{2} d \mu(g H),
$$


is well-defined (maybe $+\infty$ ) and is finite if (say) $F$ has compact support modulo $H$. The representation $I_{\zeta}$ extends as a unitary representation for this inner product.

Let $\sigma, \tau$ be two complex numbers, and consider the representations $\pi_{\sigma}$ and $\pi_{\tau}$. As $C^{\infty}(S)$ is a Fréchet space, the projective and inductive topologies on the tensor product $C(S) \otimes C(S)$ coincide, and the (completed) tensor product is realized as $C^{\infty}(S \times S)$. Hence the tensor product $\pi_{\sigma} \otimes \pi_{\tau}$ is naturally realized on $\mathcal{C}^{\infty}(S \times S)$. Explicitly,

$$
\pi_{\sigma} \otimes \pi_{\tau}(g) f\left(x_{1}, x_{2}\right)=\kappa\left(g^{-1}, x_{1}\right)^{\rho+\sigma} \kappa\left(g^{-1}, x_{2}\right)^{\rho+\tau} f\left(g^{-1}\left(x_{1}\right), g^{-1}\left(x_{2}\right)\right)
$$

for $g \in G, f \in \mathcal{C}^{\infty}(S \times S), x_{1}, x_{2} \in S$.

For $f$ in $\mathcal{C}^{\infty}(S \times S)$, let $P_{\sigma, \tau} f$ be the function on $G$ defined by

$$
\left(P_{\sigma, \tau} f\right)(g)=\kappa\left(g, \mathbf{1}^{+}\right)^{\rho+\sigma} \kappa\left(g, \mathbf{1}^{-}\right)^{\rho+\tau} f\left(g\left(\mathbf{1}^{+}\right), g\left(\mathbf{1}^{-}\right)\right)
$$

Proposition 4.2. $P_{\sigma, \tau} f$ satisfies the relation

$$
\left(P_{\sigma, \tau} f\right)(g h)=\nu_{\sigma-\tau}(h)^{-1}\left(P_{\sigma, \tau} f\right)(g),
$$

for $g$ in $G$ and $h$ in $H$.

Proof. Recall that the elements of $H$ fix both $\mathbf{1}^{+}$and $\mathbf{1}^{-}$. Moreover,

$$
\kappa\left(a_{t}, \mathbf{1}^{ \pm}\right)=e^{\mp t}, \quad t \in \mathbb{R} .
$$

Now (44) follows (6).

The map $P_{\sigma, \tau}$ will be regarded as a map from $C^{\infty}(S \times S)$ into $\mathcal{L}_{\sigma-\tau}$. Let $\sigma$ and $\tau$ be pure imaginary. Observe that

$$
\left|g\left(\mathbf{1}^{+}\right)-g\left(\mathbf{1}^{-}\right)\right|=2 \kappa\left(g, \mathbf{1}^{+}\right)^{\frac{1}{2}} \kappa\left(g, \mathbf{1}^{-}\right)^{\frac{1}{2}},
$$

so that

$$
\left\|P_{\sigma, \tau} f\right\|^{2}=2^{-2(n-1)} \int_{S \times S}|s-t|^{2(n-1)}|f(s, t)|^{2} \frac{d s d t}{|s-t|^{2(n-1)}}=2^{-2(n-1)}\|f\|^{2}
$$

and $P_{\sigma, \tau}$ extends as an isometry (up to a constant) from $L^{2}(S \times S)$ onto the space of square-integrable elements of $\mathcal{L}_{\sigma-\tau}$ for the inner product associated to (41).

Proposition 4.3. $P_{\sigma, \tau}$ intertwines the representation $\pi_{\sigma} \otimes \pi_{\tau}$ and the representation $I_{\sigma-\tau}$, i.e. for any $g$ in $G$

$$
P_{\sigma, \tau} \circ\left(\pi_{\sigma} \otimes \pi_{\tau}\right)(g)=I_{\sigma-\tau}(g) \circ P_{\sigma, \tau}
$$


Proof. Let $f$ be in $\mathcal{C}^{\infty}(S \times S)$. For $\gamma$ in $G$,

$$
\begin{gathered}
P_{\sigma, \tau}\left(\left(\pi_{\sigma} \otimes \pi_{\tau}\right)(\gamma) f\right)(g)= \\
\kappa\left(g, \mathbf{1}^{+}\right)^{\rho+\sigma} \kappa\left(g, \mathbf{1}^{-}\right)^{\rho+\tau} \kappa\left(\gamma^{-1}, g\left(\mathbf{1}^{+}\right)\right)^{\rho+\sigma} \kappa\left(\gamma^{-1}, g\left(\mathbf{1}^{-}\right)\right)^{\rho+\tau} \ldots \\
\ldots f\left(\gamma^{-1}\left(g\left(\mathbf{1}^{+}\right), g\left(\mathbf{1}^{-}\right)\right)\right) .
\end{gathered}
$$

On the other hand,

$$
\begin{gathered}
I_{\sigma-\tau}(\gamma) P_{\sigma, \tau} f(g)= \\
\kappa\left(\gamma^{-1} g, \mathbf{1}^{+}\right)^{\rho+\sigma} \kappa\left(\gamma^{-1} g, \mathbf{1}^{-}\right)^{\rho+\tau} f\left(\gamma^{-1}\left(g\left(\mathbf{1}^{+}\right), g^{-1}\left(\mathbf{1}^{-}\right)\right)\right) .
\end{gathered}
$$

The two expressions are easily seen to be equal by using (6).

\subsection{Construction of a $\left(H, \nu_{\zeta}\right)$ covariant function for $\pi_{\lambda}$}

The next step consists in finding in the representation space for $\pi_{\lambda}$ an element $\Theta_{\lambda, \zeta}$ which transforms under the action of $H$ by the character $\nu_{\zeta}$. In general, it will be a distribution on $S$ (a commun fact in harmonic analysis on semisimple symmetric space (cf [15]). We use a geometric approach through the realization of $G / H$ as $\mathcal{X}$ (see Proposition 4.1).

Let $\Pi$ be an element of $\mathcal{X}$ and $s$ be in $S$. Observe that $\Pi^{\perp}$ is a an $(n-1)$-dimensional space, complementary to $\Pi$, and the restriction of $q$ to $\Pi^{\perp}$ is negative-definite. Define

$$
\Psi(\Pi, s)=2\left(-q\left(\operatorname{proj}_{\Pi^{\perp}} \widetilde{s}\right)\right)^{\frac{1}{2}} .
$$

Proposition 4.4. Let $\left(s_{1}, s_{2}\right)$ be in $S_{\top}^{2}$. Then, for any $s_{3}$ in $S$,

$$
\Psi\left(\Pi\left(s_{1}, s_{2}\right), s_{3}\right)=2 \frac{\left|s_{1}-s_{3}\right|\left|s_{2}-s_{3}\right|}{\left|s_{1}-s_{2}\right|} .
$$

Proof. Let $\sigma_{3}=\operatorname{proj}_{\Pi \perp} \widetilde{s}_{3}$. Then there exist real numbers $\alpha_{1}$ and $\alpha_{2}$ such that $\sigma_{3}=\widetilde{s}_{3}-\alpha_{1} \widetilde{s}_{1}-\alpha_{2} \widetilde{s}_{2}$. The numbers $\alpha_{1}$ and $\alpha_{2}$ are determined by the conditions

$$
\left[\sigma_{3}, \widetilde{s}_{1}\right]=\left[\sigma_{3}, \widetilde{s}_{2}\right]=0
$$

Hence,

$$
1-s_{3} . s_{2}-\alpha_{1}\left(1-s_{1} . s_{2}\right)=\left[\widetilde{s}_{3}, \widetilde{s}_{2}\right]-\alpha_{1}\left[\widetilde{s}_{1}, \widetilde{s}_{2}\right]=\left[\sigma_{3}+\alpha_{2} \widetilde{s}_{2}, \widetilde{s}_{2}\right]=0
$$

so that

$$
\alpha_{1}=\frac{1-s_{2} \cdot s_{3}}{1-s_{1} \cdot s_{2}}=\left(\frac{\left|s_{2}-s_{3}\right|}{\left|s_{1}-s_{2}\right|}\right)^{2}
$$


and similarly

$$
\alpha_{2}=\frac{1-s_{3} . s_{1}}{1-s_{1} . s_{2}}=\left(\frac{\left|s_{1}-s_{3}\right|}{\left|s_{1}-s_{2}\right|}\right)^{2} .
$$

Now

$$
\begin{gathered}
-q\left(\sigma_{3}\right)=-\left[\widetilde{s}_{3}-\alpha_{1} \widetilde{s}_{1}-\alpha_{2} \widetilde{s}_{2}, \widetilde{s}_{3}-\alpha_{1} \widetilde{s}_{1}-\alpha_{2} \widetilde{s}_{2}\right] \\
\left.=2 \alpha_{1}\left[\widetilde{s}_{1}, \widetilde{s}_{3}\right]+2 \alpha_{2}\left[\widetilde{s}_{2}, \widetilde{s}_{3}\right]-2 \alpha_{1} \alpha_{2}\left[\widetilde{s}_{1}, \widetilde{s}_{2}\right]\right) \\
=\left(\frac{\left|s_{1}-s_{3}\right|\left|s_{2}-s_{3}\right|}{\left|s_{1}-s_{2}\right|}\right)^{2} .
\end{gathered}
$$

Proposition 4.5. Let $g$ be in $G$. Then, for any $\Pi$ in $\mathcal{X}$ and $s$ in $S$,

$$
\Psi(g(\Pi), g(s))=\kappa(g, s) \Psi(\pi, s)
$$

Proof. As $g$ preserves the form $q$ and hence the orthogonality relative to $q$, for any $\Pi$ in $\mathcal{X}$

$$
g \circ \operatorname{proj}_{\Pi^{\perp}}=\operatorname{proj}_{g(\Pi) \perp} \circ g .
$$

Hence

$$
\left.q\left(\operatorname{proj}_{g(\Pi) \perp} g(s)\right)=(g \widetilde{s})_{0}^{-2} q\left(\operatorname{proj}_{g(\Pi) \perp} g \widetilde{s}\right)=(g \widetilde{s})_{0}\right)^{-2} q\left(\operatorname{proj}_{\Pi \perp} s\right)
$$

so that

$$
\Psi(g(\Pi), g(s))=(g \widetilde{s})_{0}^{-1} \Psi(\Pi, s)=\kappa(g, s) \Psi(\pi, s)
$$

Let $\Pi_{0}=\Pi\left(\mathbf{1}^{+}, \mathbf{1}^{-}\right)=\left\{\widetilde{x}=\left(x_{0}, x_{1}, 0, \ldots, 0\right), x_{0}, x_{1} \in \mathbb{R}\right\}$, and let

$$
\Psi_{0}(s)=\Psi\left(\Pi_{0}, s\right)=\left|\mathbf{1}^{+}-s\right|\left|\mathbf{1}^{-}-s\right|=2\left(x_{2}^{2}+\cdots+x_{n}^{2}\right)^{\frac{1}{2}} .
$$

As a consequence of the previous proposition, the function $\Psi_{0}$ has a nice transformation law under the action of $H$.

Lemma 4.1. Let $h=\left(\begin{array}{ccc}\cosh t & \sinh t & 0 \\ \sinh t & \cosh t & 0 \\ 0 & 0 & k\end{array}\right)$, where $t$ is in $\mathbb{R}$, and $k$ is in $S O(n-1)$. For any $s$ in $S$

$$
\Psi_{0}(h(s))=\kappa(h, s) \Psi_{0}(s) .
$$


Let $\lambda$ and $\zeta$ be two complex numbers. For $s$ in $S$ define

$$
\Theta_{\lambda, \zeta}(s)=\Psi_{0}(s)^{-\rho-\lambda}\left|\frac{\mathbf{1}^{+}-s}{\mathbf{1}^{-}-s}\right|^{\zeta}=\left|\mathbf{1}^{+}-s\right|^{-\rho-\lambda+\zeta}\left|\mathbf{1}^{-}-s\right|^{-\rho-\lambda-\zeta} .
$$

Proposition 4.6. The function $\Theta_{\lambda, \zeta}$ satisfies

$$
\pi_{\lambda}(h) \Theta_{\lambda, \zeta}=\nu_{\zeta}(h) \Theta_{\lambda, \zeta}
$$

for all $h$ in $H$.

Proof. Let $h$ be in $H$. Then

$$
\begin{gathered}
\pi_{\lambda}(h) \Theta_{\lambda, \zeta}(s)=\kappa\left(h^{-1}, s\right)^{\rho+\lambda} \Theta_{\lambda, \zeta}\left(h^{-1}(s)\right) \\
=\kappa\left(h^{-1}, s\right)^{\rho+\lambda} \Psi_{0}\left(h^{-1}(s)\right)^{-\rho-\lambda}\left|\frac{\mathbf{1}^{+}-h^{-1}(s)}{\mathbf{1}^{-}-h^{-1}(s)}\right|^{\zeta} \\
=\kappa\left(h^{-1}, s\right)^{\rho+\lambda} \kappa\left(h^{-1}, s\right)^{-\rho-\lambda} \Psi_{0}(s)^{-\rho-\lambda}\left|\frac{\kappa\left(h^{-1}, \mathbf{1}^{+}\right)}{\kappa\left(h^{-1}, \mathbf{1}^{-}\right)}\right|^{\frac{\zeta}{2}}\left|\frac{\mathbf{1}^{+}-s}{\mathbf{1}^{-}-s}\right|^{\zeta} \\
=\Psi_{0}(s)^{-\rho-\lambda}\left(e^{2 t}\right)^{\frac{\zeta}{2}}\left|\frac{\mathbf{1}^{+}-s}{\mathbf{1}^{-}-s}\right|^{\zeta}=\nu_{\zeta}(h) \Theta_{\lambda, \zeta}(s) .
\end{gathered}
$$

by using (45).

\subsection{The duality between $I_{\zeta}$ and $\pi_{\lambda}$}

Now define the corresponding Fourier transform $\mathcal{F}_{\lambda, \zeta}$ : for $F$ a smooth section of $L_{\zeta}$ with compact support modulo $H$, define $\mathcal{F}_{\zeta, \lambda} F$ by

$$
\mathcal{F}_{\lambda, \zeta} F(s)=\int_{G / H} F(g) \pi_{\lambda}(g) \Theta_{\lambda, \zeta}(s) d \mu(g H) .
$$

Observe that, thanks to (53) the integrand is a function on $G / H$, so that the integral makes sense, the result being in general a distribution on $S$.

Proposition 4.7. For any $g$ in $G$

$$
\mathcal{F}_{\lambda, \zeta} I_{\zeta}(g)=\pi_{\lambda}(g) \mathcal{F}_{\lambda, \zeta}
$$


Proof. Set $\Theta=\Theta_{\lambda, \zeta}, \mathcal{F}=\mathcal{F}_{\lambda, \zeta}$, let $F$ be in $\mathcal{L}_{\zeta}$ with compact support modulo $H$ and let $\gamma$ be in $G$. Then

$$
\mathcal{F} \circ I_{\zeta}(\gamma) F(s)==\int_{G / H} F\left(\gamma^{-1} g\right) \kappa\left(g^{-1}, s\right)^{\rho+\lambda} \Theta\left(g^{-1}(s)\right) d \mu(g H) .
$$

Set $\gamma^{-1} g=l$ and use the invariance of the measure $d \mu$ to obtain

$$
\begin{gathered}
\mathcal{F} \circ I_{\zeta}(\gamma) F(s)=\int_{G / H} F(l) \kappa\left(l^{-1} \gamma^{-1}, s\right)^{\rho+\lambda} \Theta\left(l^{-1}\left(\gamma^{-1}(s)\right) d \mu(l H)\right. \\
=\kappa\left(\gamma^{-1}, s\right)^{\rho+\lambda} \int_{G / H} F(l) \kappa\left(l^{-1}, \gamma^{-1}(s)\right)^{\rho+\lambda} \Theta\left(l^{-1}\left(\gamma^{-1}(s)\right) d \mu(l H)\right. \\
=\kappa\left(\gamma^{-1}, s\right)^{\rho+\lambda} \int_{G / H} F(l) \pi_{\lambda}(l) \Theta\left(\gamma^{-1}(s)\right) d \mu(l H) \\
=\kappa\left(\gamma^{-1}, s\right)^{\rho+\lambda} \mathcal{F} F\left(\gamma^{-1}(s)\right)=\left(\pi_{\lambda}(\gamma) \mathcal{F} F\right)(s) .
\end{gathered}
$$

\subsection{Application to trilinear forms}

Theorem 4.1. Let $\lambda_{1}, \lambda_{2}, \lambda_{3}$ be three complex numbers. For $f_{1}, f_{2}, f_{3}$ three functions in $\mathcal{C}^{\infty}(S)$, let $T\left(f_{1}, f_{2}, f_{3}\right)$ be defined by

$$
T\left(f_{1}, f_{2}, f_{3}\right)=\left(\mathcal{F}_{-\lambda_{3}, \lambda_{1}-\lambda_{2}} P_{\lambda_{1}, \lambda_{2}}\left(f_{1} \otimes f_{2}\right), f_{3}\right) .
$$

Then, for any $g \in G$,

$$
T\left(\pi_{\lambda_{1}}(g) f_{1}, \pi_{\lambda_{2}}(g) f_{2}, \pi_{\lambda_{3}}(g) f_{3}\right)=T\left(f_{1}, f_{2}, f_{3}\right),
$$

whenever the right hand side is well defined.

Proof. Let $g$ be in $G$ and let $f_{1}, f_{2}, f_{3}$ be three functions in $\mathcal{C}^{\infty}(S)$. Then

$$
\begin{gathered}
T\left(\pi_{\lambda_{1}}(g) f_{1}, \pi_{\lambda_{2}}(g) f_{2}, \pi_{\lambda_{3}}(g) f_{3}\right)=\left(\mathcal{F}_{-\lambda_{3}, \lambda_{2}-\lambda_{1}}, P_{\lambda_{1}, \lambda_{2}}\left(\pi_{\lambda_{1}}(g) f_{1} \otimes \pi_{\lambda_{2}}(g) f_{2}\right), \pi_{\lambda_{3}}(g) f_{3}\right) \\
=\left(\mathcal{F}_{-\lambda_{3}, \lambda_{1}-\lambda_{2}} I_{\lambda_{1}-\lambda_{2}}(g) P_{\lambda_{1}, \lambda_{2}}\left(f_{1} \otimes f_{2}\right), \pi_{\lambda_{3}}(g) f_{3}\right)
\end{gathered}
$$

(use (46))

$$
=\left(\pi_{-\lambda_{3}}(g)\left(\mathcal{F}_{-\lambda_{3}, \lambda_{1}-\lambda_{2}} P_{\lambda_{1}, \lambda_{2}}\left(f_{1} \otimes f_{2}\right)\right), \pi_{\lambda_{3}}(g) f_{3}\right)
$$

(use (55))

$$
=T\left(f_{1}, f_{2}, f_{3}\right) \text {. }
$$

(use (13)). 
Making explicit the right handside of (56) shows that it coincides (up to a constant) with the former expression of $\mathcal{T}_{\boldsymbol{\lambda}}$ where $\boldsymbol{\lambda}=\left(\lambda_{1}, \lambda_{2}, \lambda_{3}\right)$ (see Theorem 2.11).

\section{Appendix : Invariant distributions supported by a submanifold}

Here is a presentation of the main results in Bruhat's theory [2, but written in terms of vector bundles and distribution densities (in the sense of [7] ch. VI). We sketch the main steps of the proof, following [17].

\section{A.1 Distribution densities for a vector bundle over a manifold}

First recall the composition of a distribution with a diffeomorphism. Let $X_{1}$ and $X_{2}$ be two open sets of $\mathbb{R}^{N}$ and let $\Phi: X_{1} \rightarrow X_{2}$ be a $\mathcal{C}^{\infty}$ diffeomorphism. Then there is a unique continous linear map $\Phi^{*}: \mathcal{D}^{\prime}\left(X_{2}\right) \rightarrow \mathcal{D}^{\prime}\left(X_{1}\right)$ which extends the composition of functions, i.e. such that $\Phi^{*} f=f \circ \Phi$ for $f \in$ $\mathcal{C}\left(X_{2}\right)$.

A distribution density on a manifold $X$ is by definition a continuous linear form on $\mathcal{C}_{c}^{\infty}(X)$. Let $u$ be a distribution density on $X$. Let $\left(X_{\kappa}, \kappa\right)$ be a local chart, i.e. $\kappa$ is a diffeomorphism of an open set $X_{\kappa}$ of $X$ onto an open set $\widetilde{X}_{\kappa}$ of $\mathbb{R}^{N}$. Then the formula

$$
u_{\kappa}(\varphi)=u(\varphi \circ \kappa)
$$

for $\varphi \in \mathcal{C}_{c}^{\infty}\left(\widetilde{X}_{\kappa}\right)$ defines a distribution $u_{\kappa}$ on $\widetilde{X}_{\kappa}$, called the local expression of $u$ in the chart $\left(X_{\kappa}, \kappa\right)$. Further, let $\left(X_{\kappa}, \kappa\right)$ and $\left(X_{\kappa^{\prime}}, \kappa^{\prime}\right)$ be two overlapping charts, and let $u_{\kappa}$ and $u_{\kappa^{\prime}}$ the corresponding local expressions of $u$. Let

$$
\Phi: \kappa^{\prime}\left(X_{\kappa} \cap X_{\kappa^{\prime}}\right) \rightarrow \kappa\left(X_{\kappa} \cap X_{\kappa^{\prime}}\right)
$$

be the change of coordinates (equal to $\kappa \circ \kappa^{\prime-1}$ ). Then

$$
u_{\kappa^{\prime}}=|\operatorname{det} d \Phi| \Phi^{*} u_{\kappa} \quad \text { in } \quad \kappa^{\prime}\left(X_{\kappa} \cap X_{\kappa^{\prime}}\right) .
$$

Conversely, suppose we have an atlas $\mathcal{F}$ of charts $\left(X_{\kappa}, \kappa\right)$ covering the manifold $M$ and suppose that for each $\kappa$ we are given a distribution $u_{\kappa} \in$ $\mathcal{D}^{\prime}\left(\widetilde{X}_{\kappa}\right)$. Assume further that for any two overlapping charts $\left(X_{\kappa}, \kappa\right)$ and $\left(X_{\kappa^{\prime}}, \kappa^{\prime}\right)$, the condition (58) is satisfied. Then the system $\left(\left(u_{\kappa}\right), \kappa \in \mathcal{F}\right)$ defines a unique distribution density $u$ on $X$ such that, for $\kappa$ in $\mathcal{F}$ and $\varphi \in \mathcal{C}_{c}^{\infty}\left(\widetilde{X}_{\kappa}\right)$ condition (57) is satisfied. 
The space of distribution densities on $X$ is denoted by $\mathcal{D}^{\prime}(X)$. A smooth density is a density the local expressions of which are $\mathcal{C}^{\infty}$ functions. The smooth densities are $\mathcal{C}^{\infty}$ sections of a line bundle called the density bundle $\Omega(X)$. It is very similar to the bundle of differential forms of maximal degree on $X$, in the sense that their transition functions just differ by an absolute value.

This definition can be extended to the case of vector bundles. Let $\pi$ : $L \rightarrow X$ be a $\mathcal{C}^{\infty}$ vector bundle over $M$, with model fiber $E_{0}$. Let $\left(X_{i}\right)$ be a family of open subsets of $X$ such that over each $X_{i}$ the bundle can be trivialized. Let $\Psi_{i}: \pi^{-1}\left(X_{i}\right) \rightarrow X_{i} \times E_{0}$ and $\Psi_{j}: \pi^{-1}\left(X_{j}\right) \rightarrow X_{j} \times E_{0}$ be two trivializations of the bundle over two overlapping subsets $X_{i}$ and $X_{j}$. Then the map $\Psi_{i} \circ \Psi_{j}^{-1}$ over $\left(X_{i} \cap X_{j}\right) \times L_{0} \rightarrow\left(X_{i} \cap X_{j}\right) \times L_{0}$ is of the form

$$
(x, v) \mapsto\left(x, g_{i j}(x) v\right)
$$

where $g_{i j}(x)$ is in $G L\left(L_{0}\right)$ and the map (transition functions of the bundle)

$$
g_{i j}: X_{i} \cap X_{j} \in x \mapsto g_{i j}(x) \in G L\left(E_{0}\right)
$$

is $\mathcal{C}^{\infty}$. A distribution density for the bundle $L$ is a system $\left(u_{i}\right)$ of distribution densities on $X_{i}$ with values in $L_{0}$ such that

$$
u_{i}=g_{i j} u_{j} \quad \text { in } X_{i} \cap X_{j} .
$$

Denote by $\mathcal{D}^{\prime}(X, L)$ the space of distribution densities for the bundle $L$.

Let $\mathcal{L}_{c}^{\infty}$ be the space of $C^{\infty}$ sections with compact support of the bundle $L$. Then the dual of $\mathcal{L}_{c}^{\infty}$ is identified with the space $\mathcal{D}^{\prime}\left(X, L^{*}\right)$. The smooth elements in the dual (those given locally by integration against a smooth function) are the $\mathcal{C}^{\infty}$ sections of the bundle $\Omega(X) \otimes L^{*}$.

\section{A.2 Invariant distribution : the case of a homogeneous vector bundle}

Let $G$ be a Lie group acting transitively on a manifold $X$. Let $o$ be a base-point in $X$, and $H=G^{o}$ be its stabilizer in $G$, so that $X \simeq G / H$. An element $h$ of $H$ acts on $X$ and fixes $o$, so that by differentiation, it acts on its tangent plane by (say) $\tau_{0}(h)$. The tangent space $T_{0} X$ can be identified with $\mathfrak{g} / \mathfrak{h}$. The element $h$ acts on $\mathfrak{g}$ by the adjoint action $\operatorname{Ad}_{\mathfrak{g}}(h)$, and preserves the subalgebra $\mathfrak{h}$ on which it acts by $\operatorname{Ad}_{\mathfrak{h}} h$. Hence its acts on $\mathfrak{g} / \mathfrak{h}$, and this action coincides with $\tau_{0}(h)$. This action satisfies

$$
\operatorname{det} \tau_{0}(h)=\frac{\operatorname{det}\left(\operatorname{Ad}_{\mathfrak{g}} h\right)}{\operatorname{det}\left(\operatorname{Ad}_{\mathfrak{h}} h\right)}
$$


The modular function $\delta_{G}$ of a Lie group $G$ is defined by

$$
\delta_{G}(g)=\left|\operatorname{det} \operatorname{Ad}\left(g^{-1}\right)\right| .
$$

so that

$$
\left|\operatorname{det} \tau_{0}(h)\right|:=\chi_{0}(h)=\frac{\delta_{H}(h)}{\delta_{G}(h)} .
$$

A homogeneous vector bundle $L$ over $X$ is a vector bundle $L$ together with an action of the group $G$ on $L$ by bundle isomorphisms. If $g$ is in $G$ and $x$ in $X$, then $g$ maps the fiber $L_{x}$ into $L_{g(x)}$ by a linear isomorphism. In particular, $H$ acts on $L_{o}$ by a representation (say) $\tau$. Conversely, given a representation $\tau$ of $H$ in a vector space $E$, then one constructs the bundle $G \times{ }_{\tau} E$ as $G \times E / \sim$, where $\sim$ is the equivalence relation defined by the right action of $H$ on $G \times E$

$$
(g, v) \sim\left(g h^{-1}, \tau(h) v\right) \quad \text { for some } h \in H,
$$

$g$ in $G$ and $v \in E$. Any homogeneous vector bundle over $X$ is of that sort, in the sense that the bundle $L$ is isomorphic to $G \times{ }_{\tau} L_{0}$ (see [16]).

A section $s: X \longrightarrow L$ can be realized as a $L_{0}$-valued function $f_{s}$ on $G$ which satisfies

$$
f_{s}(g h)=\tau^{-1}(h) f_{s}(g)
$$

and, conversely, such a function $f$ gives raise to a section of $L$. Let $\mathcal{L}_{c}^{\infty}$ be the space of smooth sections of $L$ with compact support. The space $\mathcal{L}_{c}^{\infty}$ is $G$-equivariantly isomorphic to $\mathcal{C}_{c}^{\infty}(G, H, \tau)$, the space of $\mathcal{C}^{\infty}$ functions on $G$ which satisfy (61) and have compact support modulo $H$. The group $G$ acts by left translations on $\mathcal{L}_{c}^{\infty}$, and this action is equivariant with the left action of $G$ on $\mathcal{C}_{c}^{\infty}(G, H, \tau)$.

The tangent bundle $T X$ of $X$ is an example of such a homogeneous bundle. The action of $H$ on the fiber $T_{0} X$ is $\tau_{0}$. Another important homogenous bundle is the bundle $\Omega(X)$ of densities over $X$. It is a line bundle, corresponding to the character of $H$ given by $\left|\operatorname{det}\left(\left(\tau(h)^{-1}\right)^{t}\right)\right|=\chi_{o}(h)^{-1} \quad(\mathrm{cf}$ (60) ), so that, in this context, we denote the fiber at $o$ of the bundle $\Omega(X)$ by $\mathbb{C}_{\chi_{0}^{-1}}$.

Theorem A1. There exists a non trivial invariant continous linear forms on $\mathcal{L}_{c}^{\infty}$ if and only if there exists a non trivial linear form $\xi$ on $E$ such that, for all $h \in H$

$$
\tau\left(h^{-1}\right)^{t} \xi=\chi_{0}(h) \xi
$$

More precisely,

$$
\left(\mathcal{L}_{c}^{\infty *}\right)^{G}=\mathcal{D}^{\prime}\left(X, L^{*}\right)^{G} \simeq\left(L_{0}^{*} \otimes \mathbb{C}_{\chi_{0}^{-1}}\right)^{H}
$$


Proof. (Sketch of) An element of the dual of $\mathcal{L}_{c}^{\infty}$ is a distribution density for the bundle $L^{*}$. If it is invariant by $G$, then the corresponding distribution density turns out to be smooth. Hence we are looking for a $G$-invariant smooth section of the bundle $L^{*} \otimes \Omega(X)$. But this is equivalent to an $H$ invariant element in the fiber at $o$.

\section{A.3 Invariant distribution supported in a submanifold}

Let $X$ be a manifold and $G$ a Lie group acting on $X$. Let $Q$ be an orbit of $G$ in $X$ and assume that $Q$ is closed. Let $L$ be a homogeneous vector bundle over $X$. Let $N$ be the normal bundle of $Q$ (i.e. the quotient bundle $\left.T X_{\mid Q} / T Q\right)$. Fix a base-point $o$ in $Q$, let $G_{o}=H$ be the stabilizer of $o$. Then $H$ acts on the tangent space $T_{o} X$, preserving the subspace $T_{o} Q$, and hence acts on the normal space $N_{o}=T_{o} X / T_{o} Q$ at $o$.

Let $T$ be a distribution on $Q$. Then the map

$$
\mathcal{C}_{c}^{\infty}(X) \ni \varphi \longmapsto\left(T, \varphi_{\mid Q}\right)
$$

defines a distribution on $X$, which we still denote by $T$.

Let $\mathcal{L}_{c}^{\infty}$ be the space of smooth sections of $L$ with compact support. Let $T$ be a continuous linear form on $\mathcal{L}_{c}^{\infty}$ supported in $Q$. Choose a local coordinate system on $X$

$$
\left(u_{1}, \ldots, u_{s}, v_{1}, \ldots, v_{r}\right)
$$

such that $v_{j}=0,1 \leq j \leq r$ are local equations for $Q$. The $\left(u_{i}\right)_{1 \leq i \leq s}$ form a coordinate system of $Q$ near $o$. On the other hand let $N$ be the normal bundle of $Q$. The family $\left(\frac{\partial}{\partial v_{j}}\right)_{1 \leq j \leq r}$ gives a local trivialization of the normal bundle $N$. Choose a local trivialization of the bundle $L$, and denote by $e_{1}^{*}, \ldots, e_{l}^{*}$ the corresponding coordinates on the fiber.

By Schwarz's local structure theorem for distributions supported in a vector subspace, there exists an integer $k \in \mathbb{N}$ (the local transversal order of the distribution), and for each $j, 1 \leq j \leq l$ and each multidiindex $\boldsymbol{\alpha}=$ $\left(\alpha_{1}, \ldots, \alpha_{r}\right)$ with $|\boldsymbol{\alpha}|=\alpha_{1}+\cdots+\alpha_{r} \leq k$ uniquely determined distributions $T_{\boldsymbol{\alpha}}^{j}$ on (some open subset of) $\mathbb{R}^{s} \subset \mathbb{R}^{s} \times \mathbb{R}^{r}$ such that

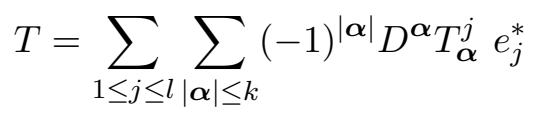

Let us consider the "top terms" subcollection $\left(T_{\boldsymbol{\alpha}}^{j},|\boldsymbol{\alpha}|=k, 1 \leq j \leq l\right)$. This collection can be interpreted as the local expression of a distribution density 
for the bundle $S^{k}(N) \otimes L_{\mid Q}^{*}$ on $Q$. This is obtained by checking the way the collection transforms under

- change of local trivialization of the bundle $L$ (change the $e_{j}^{*}$ 's)

- change of local trivialization of the normal bundle (change the $v_{j}$ 's)

- change of the local coordinate system on $Q$ (change the $u_{j}$ 's). $T$.

Denote by $\sigma^{(k)}(T)$ the section of the bundle $S^{k}(N) \otimes L_{\mid Q}^{*}$ associated to

Denote by $\mathcal{D}_{k}^{\prime}(Q, L)$ the space of continuous linear forms on $\mathcal{L}_{c}^{\infty}$ which are supported on $Q$ and of transversal order $\leq k$ on any local chart of $X$.

Theorem A2. The map $T \longmapsto \sigma^{(k)}(T)$ is a linear map from $\mathcal{D}_{k}^{\prime}(Q, L)$ in $\mathcal{D}^{\prime}\left(Q, S^{k}(N) \otimes L_{\mid Q}^{*}\right)$, with kernel $\mathcal{D}_{k-1}^{\prime}(Q, L)$. Moreover, if $\Phi$ is a bundle diffeomorphism of the bundle $L$, which maps $Q$ into $Q$, then

$$
\sigma^{(k)}\left(\Phi^{*} T\right)=\Phi^{*} \sigma^{(k)}(T),
$$

where $\Phi^{*}$ denotes the action naturally induced by $\Phi$ on $\mathcal{D}^{\prime}(Q, L)$ or of the restriction of $\Phi_{\mid L_{\mid Q}}$ on $\mathcal{D}^{\prime}\left(Q, S^{k}(N) \otimes L_{\mid Q}^{*}\right)$.

Assume now that $T$ is invariant by $G$. Obersve that invariance insures that the local transversal degree of $T$ is the same for all charts of $Q$.

Denote by $\mathcal{D}_{k}^{\prime}(Q, L)^{G}$ the space of $G$-invariant elements of $\mathcal{D}_{k}^{\prime}(Q, L)$. The conjonction of Theorem A 1 and Theorem $\mathrm{A} 2$ gives some estimate of its dimension.

Theorem A3. For any $k \in \mathbb{N}$,

$$
\operatorname{dim}\left(\mathcal{D}_{k}^{\prime}(Q, L)^{G} / \mathcal{D}_{k-1}^{\prime}(Q, L)^{G}\right) \leq \operatorname{dim}\left(\mathcal{S}_{k}\left(N_{0}\right) \otimes L_{0}^{*} \otimes \mathbb{C}_{\chi_{0}^{-1}}\right)^{H} .
$$

The theorem is mostly used in through the following corollary.

Corollary A1. Assume that for any $k \in \mathbb{N}$

$$
\left(\mathcal{S}_{k}\left(N_{0}\right) \otimes L_{0}^{*} \otimes \mathbb{C}_{\chi_{0}^{-1}}\right)^{H}=\{0\} .
$$

Then there exists no non trivial continuous $G$-invariant linear form on $\mathcal{L}_{c}^{\infty}$ such that $\operatorname{Supp}(T) \subset Q$.

\section{References}

[1] Bernstein J. and Reznikov A., Estimates of automorphic functions, Mosc. Math. J. 4 (2004), no. 1, 19-37 
[2] Bruhat F., Sur les représentations induites des groupes de Lie, Bull. S.M.F., 84, 97-205

[3] Clerc J-L., Kobayashi T., Ørsted B. and Pevzner M., Generalized Bernstein-Reznikov integrals, submitted (2009)

[4] Clerc J-L. and Neeb K-H., Orbits of triples in the Shilov boundary of a bounded symmetric domain, Transform. Groups 11 (2006), 387-426

[5] Deitmar A., Invariant triple products, Int. J. Math. Sci. (2006), art. ID 48274

[6] Gelfand I. and Shilov G., Generalized functions, vol. 1, Academic Press (1964)

[7] Hörmander L., The analysis of linear partial differential operators I, Springer Verlag (1983)

[8] Littelmann P., On spherical double cones, J. Algebra 166 (1994), 142157

[9] Loke H., Trilinear forms of $\mathfrak{g l}_{2}$, Pacific. J. Math. 197 (2001), 119-144

[10] Magyar P., Weyman J. and Zelevinsky A., Multiple flag varieties of finite type, Adv. in Math. 141 (1999), 97-118

[11] Molcanov V., Tensor products of unitary representions of the threedimensional Lorentz group, Math. USSR Izvestija, 15 (1980), 113-143

[12] Oksak A., Trilinear Lorentz invariant forms, Comm. Math. Phys. 29 (1973), 189-217

[13] Sabbah C., Polynômes de Bernstein-Sato à plusieurs variables, Séminaire Équations aux dérivées partielles (École Polytechnique), exp. 19 (1986-1987).

[14] Takahashi R., Sur les représentations unitaires des groupes de Lorentz généralisés, Bull. Math. Soc. France 91 (1963), 289-433

[15] van den Ban E., The principal series for a reductive symmetric space. I. $H$-fixed distribution vectors, Ann. Sci. cole Norm. Sup. (4) 21 (1988), no. 3, 359-412

[16] Wallach N., Harmonic analysis on homogeneous spaces, Marcel Dekker (1972)

[17] Warner G., Harmonic analysis on semi-simple Lie groups I, Springer Verlag (1972)

Addresses

(JLC) Institut Élie Cartan, Université Henri Poincaré (Nancy 1), 54506 Vandoeuvre-lèsNancy, France.

(BØ) Matematisk Institut, Byg. 430, Ny Munkegade, 8000 Aarhus C, Denmark.

jlclerc@iecn.u-nancy.fr, orsted@imf.au.dk, 